\title{
PROSODIA EMOCIONAL Y CONVERSACIÓN ESPONTÁNEA: BASES PARA EL ESTABLECIMIENTO DE UN PROTOCOLO DE IDENTIFICACIÓN PERCEPTIVA
}

\author{
Xose A. Padilla-García \\ Grupo EHSEE-Fonoemoción, Universitat d'Alacant/University of Alicante \\ xose.padilla@ua.es
}

Recibido: 30 de junio de 2020

Aceptado: 18 de noviembre de 2020

\section{Resumen}

En este artículo proponemos un protocolo metodológico que permita sortear los problemas que presenta la identificación perceptiva de las emociones en el habla espontánea. El trabajo que expondremos se divide en dos partes. La primera incluye reflexiones sobre la nómina de fenómenos emocionales, la fuente y la selección de ejemplos, la metodología de identificación de emociones, e instrucciones sobre las unidades conversacionales y prosódicas que le servirán de base. La segunda parte evalúa la guía de observación que proponemos examinando los resultados obtenidos por dos observadores en su análisis de una conversación concreta. La aplicación del estadístico kappa de Cohen a las estimaciones realizadas por los dos observadores permiten considerar favorablemente su uso como herramienta de identificación perceptiva de la prosodia emocional.

Palabras clave: emociones, prosodia, conversación coloquial

\section{Resum}

En aquest article, proposem un protocol metodològic que permet superar els problemes que presenta la identificació perceptiva de les emocions en la parla espontània. El treball que exposarem es divideix en dues parts. La primera inclou reflexions sobre els fenòmens emocionals, la font i la selecció d'exemples, la metodologia d'identificació d'emocions, i instruccions sobre les unitats conversacionals i prosòdiques que li serviran de base. La segona part avalua la guia d'observació que proposem examinant els resultats obtinguts per dos observadors en la seva anàlisi d'una conversa concreta. L'aplicació de l'estadístic kappa de Cohen a les estimacions realitzades pels dos observadors permeten considerar favorablement el seu ús com a eina d'identificació perceptiva de la prosòdia emocional.

Paraules clau: emocions, prosòdia, conversa col·loquial

\begin{abstract}
In this paper we propose a methodological protocol to avoid the problems of perceptive identification of emotions in spontaneous speech. The work is divided into two parts. The first one includes reflections about emotional phenomena, the source and selection of examples, the methodology to identify emotions, and instructions about the conversational and prosodic units on which they are based. The second one assesses the proposed observation guide by examining the results obtained by two observers in their analysis of a specific conversation. The application of Cohen's kappa statistics to the estimations carried out by two observers allows to consider favorably its use as a tool for the perceptive identification of the emotional prosody.
\end{abstract}

Keywords: emotions, prosody, colloquial speech 
En realidad, toda frase enunciativa, interrogativa o volitiva, al salir de los labios, aparece más o menos matizada por el estado de ánimo en que ha sido dicha.

(T. Navarro Tomás, 1944)

Anger, fear, aggression, the dark side are they. Once you start down the dark path forever will it dominate your destiny. (Star Wars, Master Yoda, Episode VI.)

\section{INTRODUCCIÓN ${ }^{1}$}

Las diferentes teorías que han tratado la emoción ${ }^{2}$ plantean dos mecanismos generales para abordar cómo experimentan las emociones los individuos: a) el interno, qué sucede en su interior; y b) el externo, cómo observar qué les sucede (Plutchik, 1994).

En el primer caso, se señalan dos tipos de indicios para abordar este fenómeno: 1) los cambios fisiológicos, es decir, las reacciones corporales, o indicios de excitación ('arousal') que producen variaciones en el sistema nervioso autónomo; y 2) los estados cognitivos, esto es, las señales de excitación mental, o psicológica, relacionadas principalmente con la actividad neuroquímica en ciertas regiones del cerebro (Frühhold et al., 2014).

En el segundo caso, cómo observar qué les sucede, se describen dos vías de análisis para obtener información: a) a través de lo que sienten, y explican que sienten los mismos individuos ('self-reports'), o identificación subjetiva; y b) a través de su reflejo físico, señales ('measures') en la cara, la voz (las cuerdas vocales, la velocidad de habla), etc., o identificación objetiva (excitación física).

Como indicaba acertadamente Carletta (1996), identificar perceptivamente fenómenos prosódicos supone asumir un alto grado de subjetividad. Los observadores de los fenómenos tienen que combinar técnicas aprendidas con intuiciones subjetivas y el equilibrio entre las dos fuentes de información no siempre es sencillo. Este problema de la lingüística es extrapolable, sin embargo, a muchas otras áreas de conocimiento. Sucede así, por ejemplo, cuando dos médicos examinan una radiografía de un paciente; o cuando dos meteorólogos hacen previsiones sobre el tiempo partiendo de indicios atmosféricos. Todo fenómeno susceptible de identificación por humanos tendrá, pues, un componente de subjetividad, independientemente de la disciplina que se aborde.

Dicho lo anterior, en este trabajo, vamos a intentar equilibrar la objetividad y la subjetividad que supone la observación de las emociones, asumiendo, por otra parte, que la identificación perceptiva de estos fenómenos tendrá siempre un inevitable sesgo subjetivo.

\footnotetext{
${ }^{1}$ Este trabajo se ha llevado a cabo gracias al proyecto del Ministerio de Economía, Industria y Competitividad (MINECO) El habla con significado emocional y expresivo: análisis fono-pragmático y aplicaciones (FFI2017-88310-P/MINECO).

${ }^{2}$ Una generalización importante de las diferentes propuestas permite considerar cuatro teorías principales sobre la emoción (Plutchik, 1994: 19): a) las teorías motivacionales (las emociones son respuestas/reacciones físicas innatas a estímulos externos); b) las teorías cognitivas (las emociones son estados de ánimo o estados cognitivos, pero con +-reacción); c) las teorías psicoanalíticas (las emociones son unidades no discretas (blending) guiadas por los rasgos [+-placer/+-dolor]); y d) las teorías evolutivas (las emociones son señales comunicativas que garantizan la supervivencia). Las respuestas emocionales combinan una decodificación rápida del estímulo (trigger of emotion) con patrones de comportamiento medianamente programados. La respuesta emocional, desde la propuesta evolutiva, tiene como misión aumentar el éxito de supervivencia de una determinada especie.
} 
El trabajo que presentamos se divide en dos partes. La primera incluye reflexiones sobre la nómina de categorías emocionales, la fuente y la selección de ejemplos, la metodología de identificación de emociones, e instrucciones sobre las unidades conversacionales y prosódicas que le servirán de base. La segunda parte evalúa la guía de observación que proponemos examinando los resultados obtenidos por dos observadores en su examen de una conversación concreta, aplicando para ello el estadístico kappa de Cohen a las estimaciones realizadas.

Señalamos, por último, que este artículo forma parte de un proyecto de investigación más amplio cuyo objetivo es estudiar la prosodia emocional en el habla espontánea. Por lo tanto, el trabajo que aquí exponemos debe considerarse como una fase previa (o perceptiva $^{3}$ ) antes de afrontar el estudio acústico de la prosodia emocional (Padilla, en prensa).

\section{2. ¿QUÉ EMOCIONES ANALIZAR?}

Probablemente, la primera cuestión que debe abordarse a la hora de estudiar las emociones en el habla es establecer el número de fenómenos analizables, o taxonomía de emociones.

Desde la propuesta inicial de Darwin $(1872)^{4}$, hay un cierto consenso en señalar la existencia de una lista de emociones primarias o básicas ('coarser emotions'). A pesar de este consenso, no todos los autores coinciden en su número. Ekman (1970, 1999), por ejemplo, propone seis emociones primarias: alegría, tristeza, miedo, sorpresa, enfado, asco; Plutchik (1994) aumenta su número hasta ocho: alegría, tristeza, miedo, sorpresa, enfado, asco, expectación (interés), aceptación; y Jack et al. (2014), por ejemplo, las reducen a cuatro: alegría, tristeza, sorpresa-miedo [ojos abiertos], enfadoasco [nariz arrugada].

Manteniendo la idea de las categorías básicas, algunos autores ${ }^{5}$ señalan, además, que las emociones pueden darse puras o presentarse como una mezcla ('blending') de dos o más emociones: tristeza-sorpresa, alegría-enfado, miedo-tristeza, sorpresa-asco, etc., añadiendo al problema descriptivo la posibilidad de que las emociones no sean categorías puras ${ }^{6}$.

Es lo que sucede, por ejemplo, en (1) (en negrita), en el que encontramos una mezcla de sorpresa y tristeza:

(1) 0221 A: [vale $\downarrow] /$ cinco convocatorias y resuelta que-si por ejemplo/ el examen de matemáticas $\downarrow$ que no me voy a- que es el único que no me voy a presentar $\downarrow$ que es el día treinta de este mes aparte de que ese día hay otro también/ yo no me voy a presentar porque no tengo ni profesor ni nada $\uparrow$ y-y paso $\downarrow$ ¿no?/ entonces yo esa convocatoria la pierdo $\downarrow$

\footnotetext{
${ }^{3}$ Véase t'Hart et al. (1990).

${ }^{4}$ Desde el primer trabajo de Darwin (1972), se han sucedido las investigaciones en disciplinas como la neurobiología (Hebb, 1949; Plogg, 1986; Frühholz et al. 2014), la psicología (Ekman, 1970, 1999; Scherer, 2005; Plutchik, 1994; Laukka, 2004; Matsumoto et al., 2008; Juslin et al. 2017), la lingüística (Ladd et al. 1985; Tatham y Morton, 2004; Pell et al., 2009; Cole, 2015; Garrido, 2019; etc.) y, más recientemente, la robótica (Picard et al., 2001; Cañamero, 2005; Ríos et al. 2011; Cowie, 2001; De Silva, y Chi Ng. 2009; Cowie et al., 2012; Canales et al., 2017; etc.).

${ }^{5}$ Véase Jang y Elfebein (2015).

${ }^{6}$ Las emociones, por otra parte, pueden manifestarse "desde otra emoción", dando lugar a metaemociones o emociones de segundo grado (Padilla, en prensa). Estas emociones se producen cuando los hablantes expresan 'miedo, desde la alegría', o 'asco desde la sorpresa', combinando dos situaciones aparentemente contradictorias en un mismo contexto conversacional.
} 


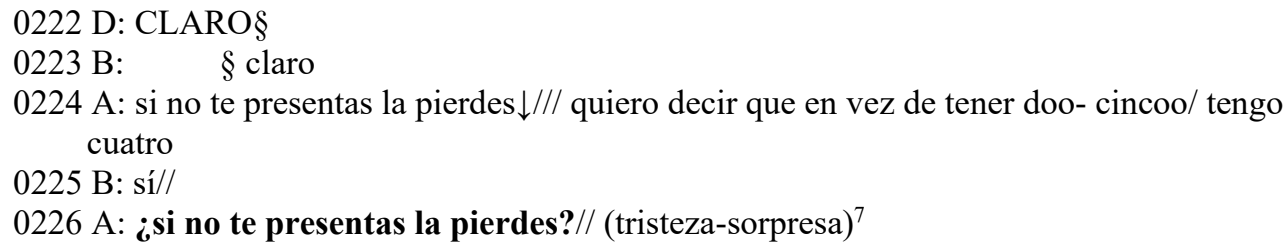

(Corpus Val.Es.Co. 2.0. [Cabedo y Pons [en línea], http://www.valesco.es])

Como alternativa, o como complemento a la propuesta de emociones primarias, los investigadores han planteado dos nuevas líneas de actuación. La primera consiste en desarrollar los subtipos de emociones, es decir, en establecer una nómina amplia de emociones secundarias (la estrella de Plutchik, 1994); y la segunda radica en extraer lo que las emociones comparten entre sí, fijando una base, como se muestra en la Figura 1, sobre la que asir las antiguas categorías: [+-] excitación (en inglés, 'arousal') y [+-] valencia ('valence'), positiva y negativa (Truesdale y Pell, 2018'; Garrido, 2019; etc).

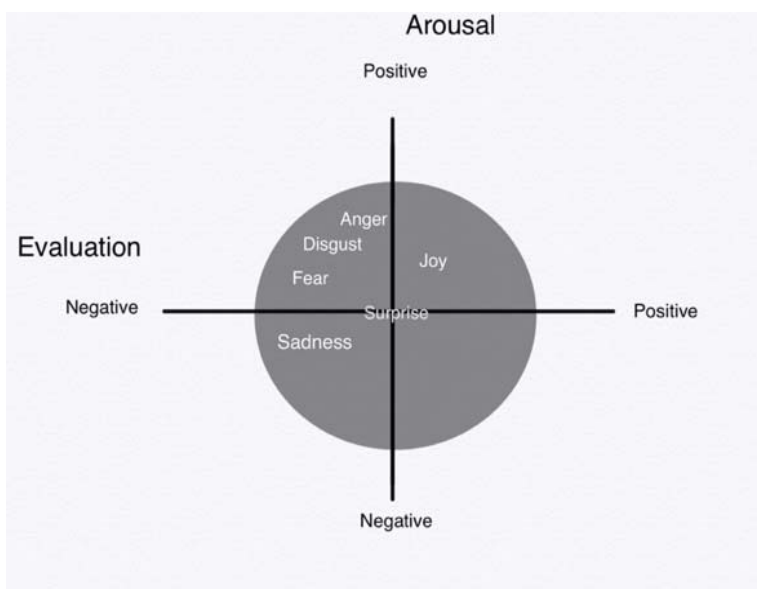

Figura 1. Distribución de las emociones básicas a lo largo del espacio arousal-evaluation (véase Garrido, 2019).

En nuestro trabajo, hemos optado por estudiar las seis emociones primarias básicas: miedo, tristeza, asco, sorpresa, enfado y alegría, señaladas por Ekman $(1970,1999)^{9}$. Comenzar por estas emociones tiene, en nuestra opinión, dos ventajas principales. La primera es que permite abordar la complejidad de un corpus coloquial -como es nuestro caso- de forma más sencilla; y, la segunda es que posibilita la agrupación de los ejemplos en categorías funcionalmente operativas, pues, su alto grado de consolidación, académica y social, facilita la labor de los analistas, sean expertos o no.

Optar por una lista cerrada de emociones no significa, lógicamente, negar la diversidad emocional, sino centrar el foco de atención en los fenómenos más fácilmente observables y establecer un punto de partida para la investigación prosódica.

\footnotetext{
${ }^{7}$ El sistema de transcripción utilizado aparece en Briz y grupo Val.Es.Co. (2002b).

${ }^{8}$ Véase también Plutchik (1994), Pell (2009), Pell et al. (2008, 2009, 2011, etc.).

${ }_{9}^{9}$ Partiendo de esta propuesta, definimos el enfado como el sentimiento de irritabilidad o indignación que experimentamos cuando somos agraviados u ofendidos; la alegría, como la sensación agradable de satisfacción y bienestar; la sorpresa, como el asombro o el malestar que provoca algo inesperado; la tristeza, como el sentimiento de desdicha o infelicidad; el miedo, como la aprehensión provocada generalmente por una sensación de amenaza, peligro, o dolor; y el asco, como la condena o intenso desagrado que nos produce algo repulsivo o repugnante.
} 


\subsection{El problema de lo neutro}

A la lista de las seis emociones señaladas, añadimos, por último, una última categoría definible como neutralidad. La neutralidad no es propiamente una emoción, sino la ausencia de la misma. Como señalan Truesdale y Pell (2018), en el centro del continuo emocional, habría un punto cero, o centro emocional ('midpoint of an emotive continuum'), que serviría de referencia en cotejos analíticos posteriores. La séptima emoción será, por tanto, como se observa en (2) en negrita, la ausencia de emoción.

(2) 0354 B: Inma ¿qué hora es?/ (neutro)

0355 A: las siete $\rightarrow$ y cuarto/ (...) (neutro)

(Corpus Val.Es.Co. 2.0. [Cabedo y Pons [en línea], http://www.valesco.es])

Atendiendo a lo anterior, un corpus coloquial no ofrecerá conversaciones exclusivamente neutras ni exclusivamente emocionales, sino espacios conversacionales neutros por oposición a los que no lo son. Existirán, consecuentemente, conversaciones que contengan más espacios neutros (actos, intervenciones, fragmentos, partes, etc.) que otras; igual que habrá conversaciones que presenten más espacios emocionales (actos, intervenciones, fragmentos, partes, etc.) que sus contrarias.

\section{EL CORPUS}

Entre las diversas opciones de corpus disponibles ${ }^{10}$, corpus espontáneos, corpus inducidos, corpus teatralizados, corpus enlatados, etc., elegir un corpus oral espontáneo para analizar las emociones parece la elección más rentable.

En la conversación coloquial espontánea, la alternancia de turnos es libre, la planificación del discurso se hace sobre la marcha $\mathrm{y}$, especialmente, la relación vivencial entre los participantes es muy intensa (Briz, 2000, 2006, 2007; Briz y grupo Val.Es.Co., 2002a, 2014). Estas características de las conversaciones coloquiales favorecerán la aparición de emociones, pero, además, proporcionarán ejemplos prosódicos alejados de todo tipo de artificio o impostación.

Nuestra fuente de ejemplos será, por consiguiente, el corpus del grupo Val.Es.Co.: Briz y grupo Val.Es.Co., 2002a y Cabedo y Pons (2013) [en línea], http://www.valesco.es. Una parte de este corpus, el Corpus Val.Es.Co. 2.0., está disponible en abierto y ofrece tanto los audios de las conversaciones espontáneas digitalizadas como las transcripciones de las mismas según el sistema propuesto en Briz y grupo Val.Es.Co. (2002b).

\section{4. ¿QUÉ UNIDADES SIRVEN DE BASE A LA OBSERVACIÓN?}

La observación perceptiva de las emociones en un corpus conversacional va a suponer ubicar la emoción hallada en unidades conversacionales concretas e indirectamente en unidades prosódicas.

La unidad conversacional más sencilla de identificar en el discurso es, lógicamente, la intervención, la unidad vinculada con el cambio de voz. Las emociones, sin embargo, pueden ocupar una intervención completa, pueden prolongarse a lo largo de varias intervenciones o pueden ser solo una parte de la misma. Consecuentemente, para ubicar

${ }^{10}$ Véase Martínez (2019). 
de manera más adecuada las emociones que encontremos, es necesario explicar brevemente qué unidades servirán de base a la observación perceptiva.

Siguiendo la propuesta del grupo Val.Es.Co. (Briz y grupo Val.Es.Co., 2002a, 2014), la intervención es, como decíamos, la unidad monológica mayor. Esta unidad conversacional se define a través de dos criterios: a) el mecanismo de reacción, es decir, las intervenciones se suceden estructuralmente unas a otras ${ }^{11}$; y b) el cambio de voz, esto es, cada intervención es responsabilidad de un emisor distinto ${ }^{12}$.

Las intervenciones están formadas a su vez por unidades más pequeñas denominadas actos (enunciados) ${ }^{13}$ (en (3), entre signos de sostenido). Estos actos, además de ser constituyentes inmediatos de la intervención, se caracterizan por dos rasgos principales: poseen fuerza ilocutiva (en nuestro caso, la emoción expresada) y tienen independencia prosódica (delimitable por fronteras más o menos precisas) ${ }^{14}$.

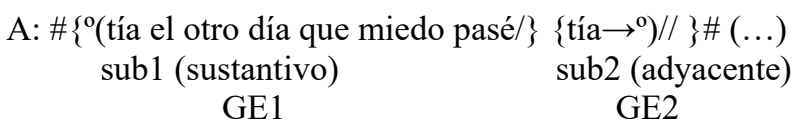

(Corpus Val.Es.Co. 2.0. [Cabedo y Pons [en línea], http://www.valesco.es])

Los actos, por otra parte, están constituidos por unidades más pequeñas llamados subactos $^{15}$ (en (3), entre llaves). Estas nuevas unidades, que son las unidades menores de la conversación, pueden tener contenido informativo, es decir, sustancia informativa (subactos sustantivos: tía el otro día que miedo pasé/), o contenido procedimental (subactos adyacentes: $t i ́ a \rightarrow{ }^{16}$ ).

Desde un punto de vista prosódico, los subactos constituyen a su vez grupos de entonación (GE), y, por lo tanto, están delimitados por pausa o inflexión (Quilis et al., 1993; Navarro Tomás, 1944). Dicho esto, es necesario aclarar, que no todos los grupos de entonación son automáticamente subactos.

En la propuesta del grupo Val.Es.Co (Briz y grupo Val.Es.Co., 2002a, 2014), se excluyen de esta categoría los reinicios (dee-, estoo-), es decir, las huellas de la

${ }^{11}$ Los hablantes, cuando conversan, pueden iniciar una intervención (intervención iniciativa), pueden reaccionar ante una intervención previa (intervención reactiva), o tienen la posibilidad de reaccionar e iniciar nuevas intervenciones (intervención reactivo-iniciativa) (Briz y grupo Val.Es.Co, 2002b y 2014; Padilla, 2019). Los conceptos iniciativo y reactivo fueron propuestos por Roulet et al. (1985).

${ }^{12}$ En los ejemplos, marcamos los diferentes emisores con letras iniciales distintas seguidas de dos puntos: A:, B:, C:, etc. En la propuesta de Val.Esco. (Briz y grupo Val.Es.Co, 2002b y 2014), todo turno de habla es intervención, pero solo las intervenciones reconocidas por el oyente de forma manifiesta y simultánea son turnos. Así, cuando la intervención de un emisor es reconocida por el oyente, el emisor adquiere el estatus de hablante.

13 Véase Briz (2002) o Briz y grupo Val.Es.Co. (2014: 39): "El acto es una unidad estructural monológica, jerárquicamente inferior a la intervención, de la que es su constituyente inmediato; asimismo, es la mínima unidad de acción e intención, que posee las propiedades de aislabilidad e identificabilidad en un contexto dado. (...) Ser aislable significa que posee fuerza ilocutiva propia y que representa una acción-intención y, por ello, tiene a menudo la capacidad de constituirse por sí mismo en una intervención en el contexto lingüístico en que aparece; no en vano es el constituyente inmediato en que puede quedar segmentada una intervención, como se ha indicado en la definición".

${ }^{14}$ Observable, igualmente, en la declinación o supradeclinación de su F0 (véase Hidalgo, 2019).

15 Aunque no tiene una equivalencia exacta, tendría relación con lo que Selkirk (1984) denomina sense units. Véase Hidalgo y Padilla (2006); Cabedo (2009).

16 El sub1 contiene también un marcador (tía), sin embargo, este marcador no es un subacto independiente, porque no hay información prosódica (ni pausa ni inflexión) que permita segmentarlo. Por esta razón segmentamos dos subactos y no tres. 
planificación conversacional del hablante. Los reinicios, en (4), en negrita, se delimitan por pausa o inflexión, pero, como se observa, no tienen contenido informativo, no son, pues, subactos:

(4) 0082 D: [ yo de lencería noo $\rightarrow$ ]/ pero si la tuviese de ropa dee- de ropa dee- [salir $\uparrow$ ]

(Corpus Val.Es.Co. 2.0. [Cabedo y Pons [en línea], http://www.valesco.es])

Partiendo de lo anterior, en un corpus conversacional, es posible encontrar, como decíamos, situaciones distintas: a) que la emoción ocupe toda la intervención (o incluso dos); b) que la emoción ocupe una parte de la intervención, normalmente, un acto (o, en su defecto, un subacto sustantivo que forme parte de un acto); y, en este último caso, c) si es un acto formado por un solo subacto, este será un grupo de entonación; si el acto está formado por varios subactos, cada subacto tendrá un grupo de entonación propio.

Lo más habitual, sin embargo, es que las emociones ocupen un acto (enunciado), pues las emociones tienen fuerza ilocutiva y suelen presentar independencia prosódica. No obstante, tomar el acto como unidad básica supone seleccionar al mismo tiempo todas las unidades monológicas menores (subacto) y mayores (intervención) que lo componen. Es decir, las unidades que manejamos son, como se muestra en la Figura 2, recursivas, ya que las unidades menores son constituyentes inmediatos de las que les preceden.

$$
\text { INTERVENCIÓN } \supset \text { ACTO } \supset \text { SUBACTO } \supset(\text { GRUPO DE ENTONACIÓN) }
$$

Figura 2. Constituyentes inmediatos de la intervención.

\section{IDENTIFICAR PERCEPTIVAMENTE UNA EMOCIÓN}

En el análisis de las conversaciones coloquiales que planteamos, y con el fin de equilibrar, como decíamos, la objetividad y la subjetividad en la identificación de las emociones, proponemos el uso de una guía de observación perceptiva. Esta guía tiene dos objetivos principales: a) ser una herramienta, o pauta facilitadora del proceso de percepción; y b) servir como una plantilla de registro que dé cuenta de los identificadores utilizados.

La guía de observación contiene ocho indicadores y, como veremos a continuación, estos indicadores están asociados a tres niveles de aproximación perceptiva: 1) el fónico-perceptivo, 2) el de las expresiones fónicas no verbales y 3) el grado de excitación.

\subsection{Primer nivel: los identificadores fónico-perceptivos}

\subsubsection{Melodía}

Atendiendo a parámetros perceptivos, y situados en el contexto emocional, usamos el término melodía ${ }^{17}$ para anotar la impresión auditiva que producen en el observador las

\footnotetext{
${ }^{17}$ La entonación, por su parte, es la interpretación lingüistica de la melodía. (Cantero, 2002: 20 y ss.).
} 
variaciones en la altura tonal o pitch (tono agudo/grave) a lo largo de la emisión del enunciado.

Desde un punto de vista retórico-pragmático, se suelen utilizar expresiones como "hablar con cierto tono, o emplear un determinado tono", y se hace referencia tanto a la pronunciación que se utiliza cuando se emite un enunciado como a la impresión que produce el mencionado tono en el receptor o destinatario del mismo (Padilla, 2004, 2017). Se engloban en este ámbito fenómenos pragmáticos y retóricos, como la ironía y la cortesía; y emociones, como la tristeza, la alegría, el enfado, etc. Señalamos en el ejemplo (5), en negrita, la parte emocional de la intervención, en este caso, sorpresa:

(5) 0290 C: [pero es un tío ¿noo?] (sorpresa)

$0128 \mathrm{~B}: \S \underline{\text { otra/ }}$ ¿qué haces tú con pasta de dientes en pantalón del pijamaa $\uparrow$ ? (sorpresa/alegría)

(Corpus Val.Es.Co. 2.0. [Cabedo y Pons [en línea], http://www.valesco.es])

La identificación perceptiva de la melodía debe verse, por otra parte, como la impresión que producen en el oyente las variaciones tonales que afectan a un enunciado completo cuando se cotejan, mental y contextualmente, los enunciados emocionales y los enunciados no emocionales (neutros).

Este cotejo se lleva a cabo tomando como punto de partida dos fuentes acústicas principales: a) las variaciones producidas en el campo tonal (el registro, el F0-range, la media de F0 del enunciado), que señalan una línea de referencia abstracta (Ladd, 1993; Gussenhoven, 2004); y b) la posición del pico inicial, la forma del cuerpo o el movimiento del tonema en el contorno melódico, que remiten igualmente a esquemas o patrones entonativos abstractos (Font-Rotchés y Cantero, 2008; Font-Rotchés, 2009).

En (6), por ejemplo, partiendo del patrón básico de la pregunta pronominal, el observador percibe un tonema descendente-ascendente, aparentemente distinto del tonema descendente esperado:

(6) 0161 B: ¿qué hilos? (enfado/sorpresa)

(Corpus Val.Es.Co. 2.0. [Cabedo y Pons [en línea], http://www.valesco.es])

\subsection{2. Énfasis}

Entendemos énfasis como el efecto perceptivo que producen en el oyente determinadas variaciones fónicas, vinculadas con fenómenos de focalización (Padilla, 2005). Estas variaciones, observables, en nuestro caso, en situaciones emocionales, pueden afectar a un sonido, a una sílaba o a una palabra completa.

Los fenómenos de énfasis han sido descritos, en general, como fenómenos fundamentalmente acentuales (Cantero, 2002), por lo tanto, estarían relacionados, en español, con el incremento de la F0, la duración, la intensidad e incluso la configuración espectral (variaciones de timbre) del sonido afectado (Gil, 2007).

Este identificador será, por tanto, un reflejo perceptivo de las modificaciones acústicas descritas como foco, foco estrecho, realce, acento enfático, etc. (Cruttenden, 1986; Ladd, 1996; Gussenhoven, 2004; etc.). 
En (7) (en negrita) se observan, por ejemplo, varias sílabas y palabras (MIEErda, síi, verGÜENza) que resultan enfáticas cuando se cotejan perceptivamente con el resto de elementos del contexto fónico en el que se enunciaron:

(7) 0244 A: ¡yo no me la voy a coger el año que viene!/ ¡una MIEErda! (enfado)

0271 B: ¡¿a que síi?! ¡a que síi!// mira yo sólo de pensar en la mirada te juro que me da escalofríos $\downarrow /$ (miedo)

0094 A: [lleva] ropa de niños $\uparrow$ y luego jerseys de punto- éste por ejemplo es de mi padre $\downarrow$ yahora os lo enseño// entonces/ pues- osea/ él como sabe e qué va $\uparrow$ y él lo compra a precio de fábrica bueno porque / él fabrica $\uparrow$ / pues tía/ cuando me voy a comprar con él- bueno he ido un par de veces $\downarrow$ pero he pasado más verGÜENza $\downarrow /$ éste es de los de mi padre $\downarrow \S$ (enfado)

(Corpus Val.Es.Co. 2.0. [Cabedo y Pons [en línea], http://www.valesco.es])

\subsubsection{Intensidad}

Entendemos la intensidad como la huella perceptiva que produce en el oyente el incremento o la disminución de la energía (número de $\mathrm{dB}$ del enunciado) en un enunciado emocional, descrito en relación con una intensidad de referencia neutra en el contexto de producción.

Atendiendo a lo anterior, podemos percibir en algunos casos un aumento general de la intensidad del enunciado, como sucede en (8), un ejemplo de enfado:

(8) $0249 \mathrm{~A}$ : por eso $\uparrow /$ yo la- yo la tengo que hacer por cojones $\rightarrow$ yo la voy a aprobar este añoß (enfado, aumento de la intensidad)

o un descenso marcado, como se advierte en (9), un ejemplo de tristeza:

(9) $0346 \mathrm{~B}$ : sí/ el año pasado aquí vino uno uuyy que era una clemencia ((...)) no se hablaba con su madree/ no se hablaba con su madree $\rightarrow$ (tristeza, disminución de la intensidad)

(Corpus Val.Es.Co. 2.0. [Cabedo y Pons [en línea], http://www.valesco.es])

\subsubsection{Velocidad de habla}

Usamos el término velocidad de habla (o de elocución) para referirnos al número de sílabas por segundo (síl. x seg.), mayor o menor, con el que se emite un enunciado (Gil, 2007).

Desde un punto de vista perceptivo, el observador atribuye una velocidad de habla (o tempo), más rápida o más lenta, a un enunciado emocional, según el contexto conversacional en el que se produjo el ejemplo, y en cotejo -como sucedía con la intensidad- con una hipotética velocidad neutra del hablante examinado en el contexto.

La velocidad de habla puede manifestarse en una parte de la intervención, es decir, en un enunciado, como en (10) (en negrita):

$(10) 0255$ A: noo tía $\rightarrow$ todo no se si voy a poder $\downarrow$ siempre quedan algunas asignaturass $\downarrow$ pero quiero decirte que-quee§ (tristeza, lenta)

o en la intervención completa, como en (11):

(11) 0296 B: ipero a ti qué más te da que sean las gafas de mentira o que sean las gafas de verdá!// i¿qué quieres que te diga que eera guapísima oo guapísimo?! ${ }^{\circ}\left(0\right.$ yo que sé) ${ }^{\circ}$ (enfado, rápida) 
La velocidad de habla, sin embargo, también puede afectar a los componentes del enunciado por separado (vocales, sílabas) produciendo alargamientos vocálicos, alargamientos consonánticos o fenómenos de silabeo.

Es lo que sucede, por ejemplo, en (12), en el que la hablante B alarga la vocal [a] y la consonante $[\mathrm{r}]$ como consecuencia, entre otras cosas, de la ralentización de la velocidad de elocución:

(12) $0229 \mathrm{~B}:=$ tendríaas tendrías que haber $\mathrm{mm}$ renunciado aa la asignatura $\rightarrow /$ anuLARRte la asignatura/ o sea como si no te hubieses matriculado// lo que pasa es que ¿tú luego te tienes que presentar en junio de esa asignatura? $/{ }^{\circ}$ (pues entonces $\left.\downarrow\right)^{\circ}$ (enfado/ velocidad lenta)

(Corpus Val.Es.Co. 2.0. [Cabedo y Pons [en línea], http://www.valesco.es])

\subsubsection{Pausas}

Describimos el identificador pausas (signos de transcripción: /, //, ///) como aquellos casos en los que los hablantes han realizado una interrupción breve del habla (involuntaria o intencional), perceptivamente relevante.

Este identificador está lógicamente relacionado con la velocidad de habla, que definimos en el apartado anterior, pues, habitualmente, la velocidad de elocución lenta va acompañada de pausas. Lo observamos, por ejemplo, en (13) (en negrita), en un ejemplo de tristeza:

(13) 0358 B: $\S$ vale/ pues a y veinticinco nos vamos ¿vale?/// y nos vamos y asíi (( ))/// que me duele un poco la cabeza $\downarrow / / /$ (tristeza, pausas largas)

(Corpus Val.Es.Co. 2.0. [Cabedo y Pons [en línea], http://www.valesco.es])

Dicho lo anterior, es necesario mencionar que no siempre hay una correlación perfecta entre velocidad y pausas. Así, es posible encontrar un enunciado, como (14), con velocidad de habla rápida, que contiene, sin embargo, dos pausas anómalas o repentinas (pragmáticamente significativas) que acentúan la tensión narrativa:

(14)0020 A: [ yo oí algo $\downarrow$ ] y / y eso/ hace mucho// psicológicamente hace mucho/ solamente de oirlo ya ( me pica) todo// total que/ que me dice tienes una cosa ahí/ y la cosa/ de repente/ pegó un salto $\uparrow$

(Corpus Val.Es.Co. 2.0. [Cabedo y Pons [en línea], http://www.valesco.es])

\subsubsection{La cualidad de la voz}

Usamos el término cualidad de la voz ('voice quality') ${ }^{18}$ para anotar la impresión perceptiva que producen en el oyente determinados ajustes fonatorios (laríngeos y supralaríngeos) que dan lugar a los llamados modos de fonación o tipos de voz: voz de 'falsetto', voz rota ('creaky voice'), voz aérea, etc. (Ladefodge, 1971; Laver, 1980; Kreiman y Sidtis, 2011; Infante, 2015; Gerellek, 2019).

Desde un punto de vista emocional, estos ajustes fonatorios (voz rota, voz aérea, voz sugerente, etc.) suponen, en ocasiones, impresiones perceptivas que pueden ayudar a identificar algunas de las emociones señaladas.

\footnotetext{
${ }^{18}$ Frente a la 'voz modal' o "normal", caracterizada por una frecuencia concreta; el 'falsetto' presenta una frecuencia más alta que la modal; la 'creaky voice' (voz rota) se caracteriza por un descenso de la F0 en comparación con la modal; etc. Tradicionalmente, hay dos posturas principales en la definición de la cualidad de la voz: la definición ancha, que incluye los estados o posturas de la glotis y el tracto vocal en general; y la definición estrecha, que se refiere a las características de la vibración de las cuerdas vocales durante la fonación de la voz (Wright et al. 2019).
} 
En (14), en negrita, encontramos, por ejemplo, un caso de voz sugerente, que suma un cierto grado de complicidad a la alegría expresada por la hablante B:

(15) 0350 B: es que estaba esperando a que le dierann lo dee la nacionalidad española $\rightarrow$ que tenía de momento un visado de seis meses en España $\rightarrow$ y ná(da) al final ya se lo dieron $\uparrow$ (( )) así quee/// ¿qué haacees? (alegría)

(Corpus Val.Es.Co. 2.0. [Cabedo y Pons [en línea], http://www.valesco.es])

En (15), por otra parte, observamos un caso de 'creaky voice', que da inicio a la elocución triste de la hablante $\mathrm{C}$ :

$(16) 0074 \mathrm{C}:(\ldots)$ porquee $\rightarrow$ para que esté siempre pará// (tristeza)

(Corpus Val.Es.Co. 2.0. [Cabedo y Pons [en línea], http://www.valesco.es])

\subsection{Segundo nivel: expresiones fónicas no verbales}

Definimos las expresiones fónicas no verbales o vocalizaciones no verbales ('nonverbal vocalisations') como aquellos elementos no verbales con correlato fónico (Sauter et al. 2010; Trouvain, 2014; Meneses y Igarashi, 2006; Kohler, 2008) que contribuyen a la identificación perceptiva de una determinada emoción.

Se incluirían, por tanto, en este grupo de rasgos: a) indicadores sonoros de reacciones fisiológicas y emocionales (risa, llanto, suspiro, carraspeo, etc.); b) elementos cuasiléxicos: interjecciones no léxicas ( $j a h$ ! ¡uy! joh!) y c) emisiones sonoras de distinto tipo: chisteos, ronquidos, uff, hm, puaf, etc. (Poyatos, 1994; Padilla, 2007; Cestero, 2019; etc.).

Encontramos un ejemplo de lo anterior en (16), en negrita, en el que las hablantes C y A utilizan las RISAS como expresión de alegría:

(17) 0008 B: [ pero/ ¿para] qué limpiaste tanto $\uparrow$ ?

0009 A: tía/ porque estaba em- para[noyá (per)día $\uparrow=]$ (alegría)

00010 C: [RISAS]

00011 A: $=[$ quee $\rightarrow]$ (entre risas) (alegría)

(Corpus Val.Es.Co. 2.0. [Cabedo y Pons [en línea], http://www.valesco.es])

u otro caso similar en (17), en el que la hablante $\mathrm{C}$ usa el bufido puf, en negrita, para reforzar la expresión de asco:

(18) 0187 A: [iqué asco de] Valencia $\uparrow$ puf! (asco)

(Corpus Val.Es.Co. 2.0. [Cabedo y Pons [en línea], http://www.valesco.es])

\subsection{Tercer nivel: grado de excitación}

Llamamos excitación a aquellas situaciones conversacionales en las que un estado de ánimo de carácter psicológico (impaciencia, nerviosismo, etc.) tiene efectos en la emisión del enunciado $\mathrm{y}$, consecuentemente, produce un reflejo perceptivo en el observador.

Aunque este rasgo afectará a varios elementos fónicos simultáneamente (F0, velocidad, pausas, etc.), lo que indicamos, en este caso, es el estado psicológico, o mental, del hablante observado. Así, este identificador, por ejemplo, permitirá diferenciar algunos ejemplos que, representando una misma emoción, contienen un grado mayor o menor de nerviosismo (agitación emocional psicológica). Todo ello, lógicamente, con efectos en la voz. 
En este sentido, es posible encontrar en el corpus ejemplos de enfado excitado [+excitación], como se observa, por ejemplo, en (19):

(19) 0296 B: ¡pero a ti qué más te da que sean las gafas de mentira o que sean las gafas de verdá!// ¿¿qué quieres que te diga que eera guapísima oo guapísimo?! ${ }^{\circ}\left(0\right.$ yo que sé) ${ }^{0}$ (enfado)

y ejemplos de enfado estático (con un bajo grado de agitación), como se advierte en (20):

(20) 0381 A: es que tía eres cabezona $\rightarrow$ y yo más aún $\uparrow$

$0382 \mathrm{C}:$ pero es que tía tú me dices $\rightarrow$ ee es un tontería $\rightarrow$ no sé por qué estamos

discutiendo por una chorrada (( )) (enfado)

$0237 \mathrm{C}:$ noo $\rightarrow$ pero no la puedes anulaar $\uparrow$ o sea $((\ldots))$ (en septiembre has perdido un convocatoria $\downarrow) \S$ (enfado)

(Corpus Val.Es.Co. 2.0. [Cabedo y Pons [en línea], http://www.valesco.es])

\subsection{Cómo utilizan la guía los observadores}

Partiendo de los identificadores que hemos señalado en el apartado anterior, los observadores examinan las conversaciones coloquiales siguiendo dos pasos: a) identificación perceptiva de la emoción mediante la audición individual de la misma y b) adjudicación de los rasgos de la guía a cada uno de los ejemplos identificados (Apéndice 1).

Como resultado del proceso anterior, es posible distinguir al menos tres situaciones distintas en relación con el número y asignación emocional de los ejemplos obtenidos: a) que el número de ejemplos total varíe entre los observadores, es decir, que uno de ellos, por ejemplo, encuentre más emociones que el otro; b) que dos observadores hayan identificado un mismo ejemplo como emocional, pero la categoría asignada sea distinta, por ejemplo, que uno llame sorpresa a lo que otro denomina miedo; y c) que los dos observadores coincidan tanto en la identificación del ejemplo como en la etiqueta emocional elegida.

Todo ello supone que, entre los observadores que examinan una conversación, puede haber un mayor o menor acuerdo o desacuerdo en la calificación de un ejemplo como emocional o no; como perteneciente o no a una determinada emoción; y como identificable o no con un número concreto de rasgos.

En los apartados siguientes, vamos a exponer, como dijimos, la evaluación de la guía de observación con un ejemplo. Es decir, examinaremos el grado de acuerdo o desacuerdo entre dos observadores que usan la guía propuesta para analizar una conversación concreta, utilizando como sistema de evaluación el estadístico kappa de Cohen.

\section{EVALUACIÓN DE LA GUÍA DE OBSERVACIÓN}

Los dos observadores de nuestro estudio examinaron la conversación BG.210.A1., perteneciente al corpus Val.Es.Co. 2002a, usando la guía que presentamos en el apartado anterior. De la observación perceptiva que llevaron a cabo, se obtuvieron dos tipos de información: a) la distinción entre intervenciones emocionales e intervenciones no emocionales y b) la identificación de 36 intervenciones emocionales (miedo, tristeza, enfado, sorpresa, alegría y asco) consensuadas (Apéndice 1), es decir, aquellas en las que los dos observadores coincidían plenamente.

En los siguientes párrafos presentaremos, por lo tanto, el resultado de cruzar las dos variables: Observador1 y Observador2, atendiendo a las situaciones que comentamos en 
el párrafo anterior. Las tablas muestran, en primer lugar, la observación más general, es decir, aquella que resulta de cotejar los datos de todas las intervenciones de la conversación conjuntamente (emocionales vs. no emocionales). Le siguen, posteriormente, la relación entre el conjunto global de emociones (tristeza, alegría, enfado, etc.) y los rasgos (melodía, énfasis, intensidad, etc.); y, por último, se presenta la evaluación de los rasgos por separado. El objetivo, como decíamos, es examinar el grado de acuerdo o desacuerdo entre los observadores a la hora de usar la guía que presentamos.

Para evaluar las estimaciones de los observadores, usaremos, como ya señalamos, el estadístico kappa de Cohen, que es la herramienta habitual en la medición del grado de acuerdo o desacuerdo entre dos observadores que examinan una misma situación ${ }^{19}$.

\subsection{Intervenciones emocionales vs. intervenciones no emocionales}

Como indicamos en el apartado 4, la intervención es la unidad conversacional monológica mayor, constituida a su vez por unidades menores: los actos (enunciados) y los subactos. Partiendo de esta definición, en este primer análisis, los observadores consideraron que una intervención era emocional si, en su totalidad, o en una de sus partes (actos o subactos), aparecía una emoción.

\subsubsection{Resultado del análisis estadístico}

Para obtener el índice kappa de Cohen $(\kappa)$, y evaluar qué grado de acuerdo presentan los dos observadores en sus análisis, hemos utilizado el software SPSS Statistics (versión 26). Este software genera dos tablas principales como 'output' del estadístico $\kappa$ : la Tabla cruzada ('Crosstabulation table') y la tabla de Medidas simétricas ('Symmetric Measures table'). En nuestro caso, las tablas generadas son: 1 y 2.

Recuento
\begin{tabular}{|l|l|r|r|r|}
\hline \multicolumn{2}{|c|}{} & \multicolumn{2}{|c|}{ Observador1 } & \multicolumn{2}{|c|}{ Total } \\
\cline { 3 - 5 } \multicolumn{2}{l|}{ Observador2 } & no emoción & emoción & \\
\cline { 2 - 5 } Total & emoción & 284 & 32 & 316 \\
66 & 37 & 382 \\
\hline
\end{tabular}

Tabla 1. Tabla cruzada Observador2*Observador1.

\begin{tabular}{|c|c|c|c|c|c|}
\hline & & Valor & $\begin{array}{c}\text { Error estándar } \\
\text { asintótico }^{\mathrm{a}}\end{array}$ & $T$ aproximada ${ }^{b}$ & $\begin{array}{c}\text { Significación } \\
\text { aproximada }\end{array}$ \\
\hline Medida de acuerdo & Kappa & ,451 &, 059 & 8,822 & 000 \\
\hline N de casos válidos & & 382 & & & \\
\hline
\end{tabular}

Tabla 2. Medidas simétricas.

a. No se presupone la hipótesis nula.

b. Utilización del error estándar asintótico que presupone la hipótesis nula.

El estadístico $\kappa$ se define habitualmente como la proporción de acuerdo o desacuerdo entre observadores que va más allá del acuerdo casual (o por suerte) (Landis y Koch, 1977). Este índice $\kappa$ puede variar entre $-1 \mathrm{y}+1$. Cuando el valor obtenido es mayor que

19 Hemos aplicado una metodología inspirada en la identificación de locutores mediante juicio de expertos. Véase Escobar y Cuervo (2008); Robles y Rojas (2015); etc. 
cero, se considera que el acuerdo no se debe a la suerte o al azar, sino que tiene relevancia estadística.

Se han proporcionado varias guías de intervalo para establecer la calidad de los acuerdos obtenidos con el índice $\kappa$. Una de las más usadas es la proporcionada por Altman (1999) ${ }^{20}$ que establece las siguientes franjas: a) un resultado es pobre ('poor') si está entre $0.00-0.20 ; b$ ) es ajustado ('fair'), si está entre $0.21-0.40 ; \mathrm{c}$ ) es moderado ('moderate'), si está entre $0.41-0.60 ; \mathrm{d}$ ) es bueno ('good'), si está entre $0.61-0.80 ; \mathrm{y}$ e) es muy bueno ('very good'), si está entre $0.81-1.00$.

La Tabla 2, que, como indicamos, examina la observación del conjunto de las intervenciones de la conversación, nos proporciona los siguientes datos: $\kappa=.451(95 \%$ CI, .335 to .566$)$ y $\mathrm{p}<.0005$.

Según la guía de Altman, un valor $\kappa$ de .451 , como el nuestro, representa un acuerdo moderado ('moderate') entre los observadores. Los datos indican, además, un valor $\mathrm{p}=.000$ (lo que significa $\mathrm{p}<.0005$ ), por consiguiente, nuestro coeficiente $\kappa$ es estadísticamente significativo y diferente de cero con un intervalo de confianza ${ }^{21}$ (CI $95 \%)$ entre .335 to .566 .

De la Tabla 1 (o tabla cruzada) obtenemos, por otra parte, que, de los 382 casos (intervenciones) evaluados por los observadores, 284 fueron calificados como no emoción, y 37 como emoción, en los ejemplos analizados. Además, hay 61 casos (i.e., $29+32)$ en el que no hubo acuerdo entre los dos observadores.

\subsection{Conjunto de intervenciones emocionales}

En este apartado examinamos si los observadores coincidían o no en el uso de los rasgos que aplicaron a las diferentes intervenciones emocionales. Recordemos que, desde un punto de vista metodológico, los observadores identifican, en primer lugar, las seis emociones reseñadas y, a continuación, registran en la plantilla (Apéndice 1) los rasgos de la guía que, según su criterio personal, han pautado su observación.

Atendiendo a lo anterior, este segundo análisis, examinamos el grado de acuerdo o desacuerdo entre los observadores atendiendo a tres situaciones: a) la aplicación de los rasgos a las intervenciones emocionales, consideradas en conjunto; b) la aplicación de los rasgos a las intervenciones emocionales, consideradas de forma individual (alegría, tristeza, miedo, etc.); y c) el uso que hicieron los observadores de cada uno de los ocho rasgos por separado como sistema de identificación general.

Del conjunto de intervenciones reseñadas en la Tabla 1 como emoción (37 casos), los observadores coincidieron, como ya indicamos, en 36: siete ejemplos de miedo, tristeza, enfado, sorpresa y alegría; y un ejemplo de asco. Es decir, en estos 36 casos, los observadores señalaron que la intervención era una emoción y que la emoción observada era la misma.

Si multiplicamos, por otra parte, los 36 ejemplos referidos por los 8 rasgos de observación (melodía, énfasis, intensidad, velocidad de habla, pausas, cualidad de voz, no verbal y excitación) obtenemos 288 casos (Apéndice 1), que evaluarán, como

\footnotetext{
${ }^{20}$ Mostramos en primer lugar la denominación de Landis y Koch (1977) y a continuación la de Altman (1999): $0.00-0.20$ Slight/ Poor; $0.21-0.40$ Fair/ Fair; $0.41-0.60$ Moderate/ Moderate; $0.61-0.80$ Substantial/ Good; 0.81 - 1.00 Almost / Very good.

${ }^{21}$ El intervalo de confianza (95\% CI) se obtiene multiplicando Z $(=1.96)$ x el error estándar (SE), y sumando y restando posteriormente esta cifra a $\kappa$ (Field, 2009: 45).
} 
veremos a continuación, la relación entre la emoción observada y los rasgos que la definen.

\subsubsection{La relación entre el conjunto global de emociones y los rasgos}

De nuevo, el software SPSS Statistics (versión 26) genera dos tablas principales como 'output' del estadístico $\kappa$ (Tablas 3 y 4):

Recuento
\begin{tabular}{|ll|r|r|r|}
\hline \multirow{2}{*}{ Observador2 } & \multicolumn{2}{|c|}{ Observador1 } & \multicolumn{2}{|c|}{ Total } \\
\cline { 3 - 5 } & rasgo ausente & 127 & 21 & 148 \\
\cline { 2 - 5 } & rasgo presente & 27 & 113 & 140 \\
\hline Total & & 154 & 134 & 288 \\
\hline
\end{tabular}

Tabla 3. Tabla cruzada Observador $2 *$ Observador 1

\begin{tabular}{|c|c|c|c|c|}
\hline & Valor & $\begin{array}{l}\text { Error estándar } \\
\text { asintótico }^{\mathrm{a}}\end{array}$ & $T$ aproximada ${ }^{b}$ & $\begin{array}{c}\text { Significación } \\
\text { aproximada }\end{array}$ \\
\hline Medida de acuerdo & ,666 & ,044 & 11,313 &, 000 \\
\hline N de casos válidos & 288 & & & \\
\hline
\end{tabular}

a. No se presupone la hipótesis nula.

b. Utilización del error estándar asintótico que presupone la hipótesis nula.

Tabla 4. Medidas simétricas

La Tabla 4 nos proporciona los siguientes datos: $\kappa=.666$ (95\% CI, .579 to .752$), \mathrm{p}<$ .0005 . Según la guía de Altman (1999), un valor $\kappa$ de .666, como el nuestro, representa un buen acuerdo ('good/substantial') entre los observadores. La Tabla 4 muestra, además, un valor $\mathrm{p}=.000$ (lo que significa $\mathrm{p}<.0005$ ), por consiguiente, el coeficiente $\kappa$ obtenido es estadísticamente significativo y diferente de cero con un intervalo de confianza (CI 95\%) entre .579 y .752.

De la tabla cruzada (Tabla 3) obtenemos, por otra parte, que de los 288 rasgos evaluados (i.e., 36 ejs. x 8 rasgos) por los observadores, 127 fueron calificados como ausentes (no aparecían en la plantilla de observación) y 113 como presentes (sí aparecían) en los ejemplos analizados. Además, hay 48 casos (i.e., 27+21) en el que no hubo acuerdo entre los observadores.

\subsubsection{La relación entre las emociones individuales y los rasgos}

SPSS Statistics genera nuevamente dos tablas principales como output del estadístico kappa de Cohen ( $\kappa)$. En este caso, dos tablas por cada una de las seis emociones consideradas de forma individual (Apéndice 2). Basándonos en la guía de Altman (1999), volvemos a encontrar un buen acuerdo ('good/substantial') entre los dos observadores en las seis emociones examinadas. Encontramos un valor $\kappa=.674(95 \%$ CI, .478 to .870), $\mathrm{p}=.000$, en el caso del miedo; $\kappa=.639$ (95\% CI, .619 to .840$)$, $\mathrm{p}=.000$, en el caso de la tristeza; $\kappa=.607(95 \% \mathrm{CI}, .40$ to .814$), \mathrm{p}=.000$, en el caso del enfado; $\kappa=.747(95 \% \mathrm{CI}, .573$ to .921$), \mathrm{p}=.000$, en el caso del sorpresa; $\kappa=.643(95 \%$ CI, .447 to .839), $\mathrm{p}=.000$, en el caso del alegría; $\kappa=.750$ (95\% CI, .442 to 1.192 ), $\mathrm{p}=.000$, en el caso del asco.

Todos ellos indican un buen acuerdo entre los observadores $\mathrm{y}$ todos son estadísticamente significativos y diferentes de cero, pues $\mathrm{p}=.000(\mathrm{p}<.0005)$. Nótese, 
sin embargo, que hay intervalos (95\% CI) más anchos y más estrechos, consecuentemente, no todos los ejemplos de la muestra ('sample') representan de igual forma la media verdadera o real ('true mean of the population').

\subsubsection{Evaluación de los rasgos por separado}

Examinamos, por último, qué sucedía con los rasgos individuales que sirvieron para identificar las emociones usando la guía (Apéndice 3).

Basándonos de nuevo en la guía de Altman (1999), encontramos un acuerdo muy bueno, casi perfecto ('very good') en los rasgos cualidad de voz con $\kappa=1$ (95\% CI, -1 to 1$), \mathrm{p}=.000$, y no verbal con $\kappa=1(95 \% \mathrm{CI},-1$ to 1$), \mathrm{p}=.000)$; un acuerdo bueno ('good') en los rasgos énfasis con $\kappa=.774$ (95\% CI, .571 to .977), $\mathrm{p}=.000$, pausas con $\kappa=.705,(95 \% \mathrm{CI}, .439$ to .971$), \mathrm{p}=.000)$; un acuerdo moderado ('moderate') en velocidad de habla con $\kappa=.462(95 \% \mathrm{CI}, .123$ to .801$), \mathrm{p}=.005$; un acuerdo ajustado ('fair') en los rasgos intensidad, con $\kappa=.341$ (95\% CI, .034 to .648), $\mathrm{p}=.017 \mathrm{y}$ excitación con $\kappa=.289$ (95\% CI, .095 to .483$), \mathrm{p}=.014)$; y un acuerdo pobre ('poor') en el rasgo melodía, $\kappa=.138(95 \% \mathrm{CI}, .108$ to .348$), \mathrm{p}=.102)$. Aunque este último dato, como muestra el valor $\mathrm{p}$, no es estadísticamente significativo.

En este tercer análisis, los rasgos muestran un comportamiento desigual. Los datos señalan una tendencia al acuerdo entre los dos observadores, ahora bien, los intervalos de confianza (CI 95\%) no siempre son estrechos, por lo tanto, no es posible afirmar, en determinados casos, que las cifras obtenidas encajen dentro de la media verdadera o real.

\section{DISCUSIÓN}

En los apartados precedentes, hemos usado el estadístico kappa de Cohen ( $\kappa)$ para determinar el grado de acuerdo o desacuerdo entre dos observadores en el uso de la guía de observación perceptiva a la hora de identificar las emociones en la conversación examinada.

Consideradas las dos variables: Observador1 y Observador2, hemos presentado la observación más general, es decir, aquella que resulta de los datos de todas las intervenciones de la conversación conjuntamente; y hemos mostrado igualmente el grado de acuerdo o desacuerdo en el uso de los identificadores de la guía en las 36 intervenciones emocionales consensuadas.

Partiendo de los niveles establecidos por Altman (1999), que propone franjas (de pobre a muy bueno) para describir el nivel de acuerdo entre los observadores, es posible señalar que hay un grado de acuerdo importante en los juicios perceptivos de los dos observadores: $\kappa=.451(95 \% \mathrm{CI}, .335$ to .566$), \mathrm{p}<.0005$. para las intervenciones; $\mathrm{y} \kappa=$ $.666(95 \% \mathrm{CI}, .300$ to .886$), \mathrm{p}<.0005$ para las 36 intervenciones emocionales. Esto último se observa, especialmente, tanto en las emociones analizadas de forma general como en las emociones examinadas de forma individual. Si bien, en este último caso, no todos los ejemplos analizados muestran un intervalo de confianza estrecho (CI 95\%).

Hay que señalar, por otra parte, que el grado de acuerdo es menor cuando examinamos los rasgos por separado. Encontramos identificadores en los que hay un acuerdo perfecto, como cualidad de voz y no verbal; otros en los que el acuerdo es bueno, como énfasis y pausas; y otros en los que el acuerdo es débil o pobre, como excitación y melodía. Llama la atención que sea, precisamente, la melodía el rasgo que presenta el 
resultado más pobre. Los intervalos de confianza (CI 95\%), en este caso, también son desiguales.

Es posible señalar, en este sentido, que los dos observadores que han participado en nuestro estudio han encontrado, quizás, algunas dificultades en la aplicación de los rasgos melodía y [+-excitación]. Probablemente, el acuerdo pobre, en el caso de la melodía, se deba hipotéticamente a que los observadores no han sabido distinguir bien entre los rasgos melodía y énfasis, que la guía diferenciaba por su ámbito de aplicación, más estrecho en el caso del énfasis, y más ancho, en el caso de la melodía. Esto nos lleva a considerar la posibilidad de fusionar estos dos rasgos en el futuro para facilitar la labor de los observadores, pues ambos rasgos, en última instancia, son informados por el tono.

Algo similar a la melodía sucede en el caso de la [+-excitación], que hemos descrito como un rasgo de tipo psicológico o mental, informado por una suma de elementos fónicos y cognitivos. Es probable quizás que los observadores no hayan podido distinguir bien, en este caso, los diferentes matices que el rasgo supone, o que el sesgo subjetivo influya demasiado en su aplicación. Consecuentemente, en el futuro, podríamos plantearnos quizás modificar su estatus dentro de la guía o incluso reconsiderar su cabida definitiva.

A pesar de los problemas que plantean los dos rasgos mencionados, los datos del análisis estadístico indican, en general, que, en esta pequeña cala, el grado de acuerdo entre los observadores parece adecuado. Ello sustentaría de algún modo que la guía de observación perceptiva parece funcionar, al menos, en el ejemplo presentado.

Dicho lo anterior, es necesario señalar, para no conducir a equívoco, que un grado de acuerdo importante entre los observadores no significa necesariamente que sus decisiones sean correctas. Es posible, por ejemplo, que ambos estén diagnosticando erróneamente un ejemplo o atribuyendo rasgos no adecuados. Lamentablemente, el índice kappa de Cohen no puede medir esta hipotética situación.

\section{CONCLUSIONES}

En este artículo hemos sentado las bases para un protocolo metodológico que permita sortear los problemas que presenta la identificación perceptiva de emociones en el habla espontánea.

Comenzamos el artículo examinando los problemas que plantea el objeto de estudio, atendiendo al número de fenómenos analizables y a las dificultades que supone su distinción. Concluido este paso, hemos expuesto el protocolo seguido para construir una guía de observación perceptiva. Hemos descrito los criterios utilizados y hemos definido los diferentes identificadores o rasgos que la componen. La guía contiene tres niveles de identificación: el fónico-perceptivo, el de las expresiones fónicas no verbales y el grado de excitación.

La segunda parte del artículo presenta una primera evaluación de la guía de observación a través del examen de un caso. En esta parte, presentamos los resultados derivados de analizar el grado de acuerdo o desacuerdo entre dos observadores usando para ello el índice kappa de Cohen.

El análisis estadístico revela que, en general, existe un buen grado de acuerdo entre los observadores evaluados. Ello se observa en las dos situaciones examinadas, esto es, en la diferenciación entre intervenciones emocionales y no emocionales y en el uso de 
rasgos para describir las 36 intervenciones emocionales consensuadas. No obstante, como indicamos en el apartado anterior, los observadores han mostrado poco acuerdo en algunos de los rasgos, como la melodía y la [+-excitación]. Esto nos lleva a pensar en la posibilidad de fusionar en el futuro los rasgos énfasis y melodía, pues ambos son informados por el tono; y en reformular quizás el estatus del rasgo [+-excitación], que bascula entre lo fónico y lo psicológico, reconsiderando incluso su cabida definitiva dentro de la guía.

A pesar de lo anterior, como hemos ido viendo en los análisis, los resultados parecen apuntar, en general, a que la guía perceptiva presentada, aunque provisional, puede ser considerada una herramienta útil como protocolo de actuación en el estudio de la prosodia emocional.

\section{REFERENCIAS BIBLIOGRÁFICAS}

Altman, Douglas G. (1999): Practical statistics for medical research. New York: NY Chapman $\&$ Hall/CRC Press.

Briz, Antonio (2000): «Turno y alternancia de turno en la conversación». Revista Argentina de Lingüistica, 16, 3-27.

Briz, Antonio (2002): «Las unidades de la conversación: el acto». Estudios ofrecidos al profesor J.J. De Bustos Tovar. Madrid: Universidad Complutense, 953-968.

Briz, Antonio (2006): «La segmentación de una conversación en diálogos», Oralia, 9, 45-72.

Briz, Antonio (2007): «La unidad superior del discurso (conversacional): el diálogo». Luis Cortés, A. Bañón, M. M. Espejo y J. L. Muñío (coords.) Discurso y oralidad. Homenaje al profesor José Jesús de Bustos Tovar. Almería: Anejo 3/1 de la Revista Oralia, 15-40.

Briz, Antonio y grupo Val.Es.Co. (2002a): Corpus de conversaciones coloquiales. Madrid: Arco Libros.

Briz, Antonio y grupo Val.Es.Co. (2002b): «El sistema de transcripción del Grupo Val.Es.Co.». Español Actual, 77, 35-55.

Briz, Antonio y grupo Val.Es.Co. (2014): «Las unidades del discurso oral. La propuesta Val.Es.Co. de segmentación de la conversación (coloquial)». Estudios de Lingüistica del Español, 35.1, 11-71.

Cabedo, Adrián. y Salvador Pons (eds.) (2013): Corpus Val.Es.Co. 2.0. Consultado online en http://www.valesco.es.

Cabedo, Adrián (2009): La segmentación prosódica en español coloquial. Valencia: Quaderns de Filologia de la Universitat de València.

Canales, Lea, Boldrini, Ester, Daeleman, Walter y Patricio Martínez (2017): «Towards the Improvement of Automatic Emotion Pre-annotation with Polarity and Subjective Information». Proceedings of Recent Advances in Natural Language Processing 4-6, 157 163.

Cantero, Francisco J. (2002): Teoría y análisis de la entonación. Barcelona: Edicions Universitat de Barcelona.

Cañamero, Lola (2005): «Emotion understanding from the perspective of autonomous robots research». Neural Networks, 18 (4), 445-455.

Carletta, Jean (1996): «Assessing agreement on classification tasks: The kappa statistic». Computational Linguistics, 22(2), 249-254.

Cestero, Ana M. (2019): «Comunicación no verbal», en M. Elena Placencia y Xose A. Padilla (eds.), Guía práctica de pragmática del español, London: Routledge, 56-65.

Cruttenden, Alan (1986): Intonation. Cambridge: Cambridge University Press.

Cole, Jennifer (2015): «Prosody in context: A review». Language, Cognition and Neuroscience, 30:1-2, 1-31.

Cowie, Roddy (2001): «Describing the emotional states expressed in speech». ISCA ITRW Speech and Emotion, Newcastle, Northern Ireland, 5, 45-56. 
Cowie, Roddy, McKeewn, Gary y Douglas-Cowie, Ellen. 2012. "Tracing Emotion: An Overview». International Journal of Synthetic Emotions, 3(1), 1-17.

Darwin, Charles (1972): The Expression of the Emotions in Man and Animals. Londres: John Murray.

De Silva, Liyanage Charles y Pei Chi Ng (2009): «Bimodal emotion recognition». Available from https://www.researchgate.net/publication/3845464_Bimodal_emotion_recognition [accessed Apr 09 2020].

Ekman, Paul (1970): «Universal Facial Expressions of Emotions». California Mental Health Research Digest, 8(4), 151-158.

Ekman, Paul (1999): «Basic emotions». T. Dalgleish and M. Power. eds. Handbook of Cognition an Emotion. New York: John Wiley \& Sons Ltd.

Escobar-Pérez, Jasmine y Ángela Cuervo-Martínez (2008): «Validez de contenido y juicio de expertos: una aproximación a su utilización». Avances en Medición, 6, 27-36.

Field, Andy (2009): Discovering Statistics Using SPSS. London: SAGE Publications.

Font-Rotchés, Dolors (2009): «Les interrogatives pronominals del català central. Anàlisi melódica i patrons entonatius». Els Marges 87, 41-64.

Font-Rotchés, Dolors y Francisco Cantero (2008): «La melodía del habla: acento, ritmo y entonación». Eufonía, 43, 19-39.

Frühholz, Sascha, Wiebke Trost y Didier Grandjean (2014): «The role of the medial temporal limbic system in processing emotions in voice and music». Progress in Neurobiology, 123:1-17. DOI:10.1016/j.pneurobio.2014.09.003

Garrido, Juan María (2019): «Análisis de las curvas melódicas del español en el habla emotiva simulada». Estudios de Fonética Experimental, X, 205-55.

Gerellek, Marc (2019): «The phonetics of voice». William Katz and Peter Assmann (eds.) The Routledge Handbook of Phonetics, London: Routledge, 1-48.

Gil, Juana (2007): Fonética para profesores de español: de la teoría a la práctica. Madrid: Arco Libros.

Gussenhoven, Carlos (2004): The phonology of tone and intonation. Cambridge: Cambridge University Press.

Hebb, Donald O (1949): The Organization of Behaviour. New York: John Wiley \& Sons.

Hidalgo, Antonio (2019): Sistema y uso de la entonación en español hablado. Aproximación interactivo-funcional. Santiago de Chile: UAH.

Hidalgo, Antonio y Xose A. Padilla (2006): «Bases para el análisis de las unidades menores del discurso oral: los subactos». Oralia, 9, 109-139.

Infante, Patricia (2015): «¿Son distintos el creak y la voz creaky?: Estudio perceptivo preliminar». RSEL, 45/1, 105-129

Jack, Rachel E., Oliver Garrod y Philippe G. Schyn (2014): «Dynamic Facial Expressions of Emotion Transmit an Evolving Hierarchy of Signals over Time». Current Biology 24, $187-$ 192.

Jang, Daisung y Hillary Elfebein (2015): «Emotion, Perception and Expression of». James David Wright (editor-in-chief), International Encyclopedia of the Social \& Behavioral Sciences, 2nd edition, Oxford: Elsevier, 7, 483-489.

Juslin, Patrik N., Laukka, Petri y Bänziger, Tania (2017): "The Mirror to Our Soul? Comparisons of Spontaneous and Posed Vocal Expression of Emotion». Journal of Nonverbal Behavior 42: 1-40.

Kohler, Klaus J. (2008): “'Speech-smile','speech-laugh','laughter'and their sequencing in dialogic interaction». Phonetica, 65(1-2), 1-18).

Kreiman, Jody, y Sidtis, Diana (2011): Foundations of Voice Studies. Oxford: Wiley-Blackwell.

Ladefoged, Peter N. (1971): Preliminaries to linguistic phonetics. Chicago: University of Chicago.

Ladd, Robert, Kim E. A. Silverman, Frank Tolkmitt, Günther Bergmann, and Klaus R. Scherer (1985): «Evidence for the independent function of intonation contour type, voice quality, 
and F0 range in signaling speaker affect». Journal of the Acoustical Society of America 78, 435.

Ladd, Robert (1993): «On the Theoretical Status of the Baseline in Modelling Intonation». Language and Speech 36(4), 435-451.

Ladd, Robert, (1996): Intonational phonology. Cambridge: Cambridge University Press.

Landis, J. Richard, \& Koch, Gary G. (1977): «The measurement of observer agreement for categorical data». Biometrics, 33, 159-174.

Laukka, Petri (2004): Vocal expression of emotion, discrete-emotions and dimensional accounts. Uppsala: Uppsala University.

Laver, John (1980): The Phonetic Description of Voice Quality. Cambridge: Cambridge University Press.

Martínez, Diana (2019): La expresión de la ironía en la conversación. Estudio fonopragmático en un corpus de habla semiespontánea. Valencia: Universitat de València.

Matsumoto David. Yoo Seung H., Fontaine Johnny (2008): «Mapping expressive differences around the world: the relationship between emotional display rules and individualism versus collectivism». J. Cross. Cult. Psychol. 39, 55-74.

Menezes, Caroline y Igarashi, Yosuke (2006): «The speech laugh spectrum». Proc. Speech Production, Brazil, 157-164.

Navarro Tomás, Tomás (1974): Manual de entonación española. Madrid: Guadarrama. (1 $\mathrm{ed}$. 1944).

Padilla, Xose A. (2004): «El tono irónico: estudio fonopragmático», Español Actual. 81, 85-94.

Padilla, Xose A. (2005): Pragmática del orden de palabras. Alicante: Universitat d'Alacant.

Padilla, Xose A. (2007): La comunicación no verbal. Madrid: Liceus.

Padilla, Xose A. (2017): «Prosodia y (des)cortesía en contexto de diálogo: la creación y la negociación del ámbito tonal». LEA, Vol. XXXIV/2, 243-268.

Padilla, Xose A. (2019): «El papel del mecanismo de reacción en la definición de las unidades conversacionales. Su contribución a la caracterización del dinamismo discursivo». Antonio Hidalgo y Adrián Cabedo. eds. Pragmática del español hablado: hacia nuevos horizontes. Valencia: Universitat de València.

Padilla, Xose A. (in press). «La voz como reacción emocional: de qué nos informa la prosodia», Spanish in Context (2021).

Pell, Marc D. 2009. «Recognizing Emotions in a Foreign Language». Journal of Nonverbal Behavior 33: 107-120.

Pell, Marc D., y Skorup, Vera. (2008): «Implicit processing of emotional prosody in a foreign versus native language». Speech Communication 50: 519-530.

Pell, Marc D., Silke Paulmanna, Chinar Daraa, Areej Alasseria, Sonja A. Kotz. (2009): "Factors in the recognition of vocally expressed emotions: A comparison of four languages». In Journal of Phonetics 37: 417-435.

Pell, Marc D. Abhishek Jaywant, Laura Monetta \& Sonja A. Kotz. (2011): «Emotional speech processing: Disentangling the effects of prosody and semantic cues». Cognition \& Emotion, 25/5: 834-853.

Picard, Rosalind W., Elias Vyzas and Jennifer Healey (2001): «Toward machine emotional intelligence: analysis of affective physiological state". IEEE Transactions on Pattern Analysis and Machine Intelligence 23/10: 1175-1191.

Plogg, D. (1986): «Biological foundations of vocal expressions of emotions». Robert Plutchik and Henry Kellerman (eds.) Biological Foundations of Emotion: Theories, Research and Experience, New York: Academic Press, 73-85.

Plutchik, Robert (1994): Emotion: Theory, research, and experience: Vol. 1. Theories of emotion, 1, New York: Academic.

Poyatos, Fernando (1994): La comunicación no verbal. 3 volúmenes. Madrid: Istmo.

Ríos, David, García, Diego, Gómez, Pablo y Alberto Redondo (2011): «Máquinas que perciben, sienten y deciden». Rev. R. Acad. Cienc. Exact. Fís. Nat. 105, 99-106.

Robles Garrote, Pilar y Manuela del Carmen Rojas (2015): «La validación por juicio de expertos: dos investigaciones cualitativas en Lingüística aplicada». RNLA 6, 4-17. 
Roulet, Edy et al. (1985): L'articulation du discours en français contemporain, Berna: Peter Lang.

Quilis, Antonio, Margarita Cantarero y Manuel Esgueva (1993): «El grupo fónico y el grupo de entonación en español hablado». RFE, 73: 55-64.

Sauter, Disa A., Eisner, Frank, Calder, Andrew J., y Scott, Sophie K. (2010): «Perceptual cues in nonverbal vocal expressions of emotion». Quarterly Journal of Experimental Psychology, 63(11), 2251-2272.

Selkirk, E. (1984): Phonology and syntax: The relation between sound and structure. Cambridge, MA: The MIT Press.

Scherer, K. et al. (2005): «Proposal for exemplars and work towards them: Theory of emotions». HUMAINE deliverable D3e http://emotion- research.net/deliverables.

t'Hart, J. T., R. Collier y A. Cohen (1990): A perceptual Study of Intonation. Cambridge: Cambridge University Press.

Tatham, Mark y Morton, Katherine (2004): Expression in Speech: Analysis and Synthesis. Oxford: Oxford U. Press.

Trouvain, Jürgen (2014): «Laughing, breathing, clicking-The prosody of nonverbal vocalisations». Proceedings of Speech Prosody, 7, 598-602.

Truesdale, Deirdre M. and Marc D. Pell (2018): «The sound of Passion and Indifference». Speech Communication 99, 124-134.

Wright, Richard, Courtney Mansfield y Laura Panfili (2019): «Voice quality types and uses in North American English», Anglophonia 27, 1-20. 


\section{APÉNDICE 1}

\begin{tabular}{|c|c|c|}
\hline MIEDO (7) & Observador1 & Observador2 \\
\hline \multicolumn{3}{|c|}{\begin{tabular}{l|l|l} 
7C: $\quad$ ¡AYY! & \\
9C: ¿¡no me digas que son las cartas esas de hacer copias/ y- y rollos de & \\
esos!?
\end{tabular}} \\
\hline Melodía 1 & $\mathrm{X}$ (agudo) & X (agudo) \\
\hline Énfasis 2 & X (jayy!) & \\
\hline Intensidad 3 & $\mathrm{X}$ (alto) & $\mathrm{X}$ (alto) \\
\hline Velocidad de habla 4 & X (rápida) & X (rápida) \\
\hline \multicolumn{3}{|l|}{ Pausas 5} \\
\hline \multicolumn{3}{|l|}{ Cualidad de la voz 6} \\
\hline Vocalizaciones no verbales 8 & X (jayy!) & X (¡ayy!) \\
\hline Excitación 7 & $\mathrm{X}$ & \\
\hline \multicolumn{3}{|l|}{$\begin{array}{l}16 C \S ; a h ! / \text { ¿UYY hace tiempo/ y yo tenía miedo de no hacerlo porque } \\
\text { eso// por si pasaba algo }\end{array}$} \\
\hline Melodía & X (agudo) & X (agudo) \\
\hline Énfasis & $\mathrm{X}\left({ }_{\mathrm{U} U Y Y} \mathrm{UY} !\right)$ & $X(i U Y Y !)$ \\
\hline Intensidad & $\mathrm{X}$ (alto) & $\mathrm{X}$ (alto) \\
\hline Velocidad de habla & X (rápida) & X (lenta) \\
\hline \multicolumn{3}{|l|}{ Pausas } \\
\hline \multicolumn{3}{|l|}{ Cualidad de la voz } \\
\hline Vocalizaciones no verbales & X (UYY) & X (UYY) \\
\hline \multicolumn{3}{|l|}{ Excitación } \\
\hline \multicolumn{3}{|l|}{$\begin{array}{l}\text { 20B: poh aquí no se ha hecho y yo no lo sé//l digo se lo vamoh a dar que } \\
\text { lo vean ellah qué dicen }\end{array}$} \\
\hline Melodía & $\mathrm{X}$ & \\
\hline \multicolumn{3}{|l|}{ Énfasis } \\
\hline Intensidad & & $\mathrm{X}$ (baja) \\
\hline Velocidad de habla & & X (rápida) \\
\hline Pausas & $\mathrm{X}(/ / /)$ & \\
\hline \multicolumn{3}{|l|}{ Cualidad de la voz } \\
\hline \multicolumn{3}{|l|}{ Vocalizaciones no verbales } \\
\hline \multicolumn{3}{|l|}{ Excitación } \\
\hline \multicolumn{3}{|l|}{$\begin{array}{l}\text { 381C: creo que dos o tres años///(6') si no pagas luego de } \\
\text { mantenimiento y dee- } ; \text { uff } \uparrow / \text { un palo!///(7') ¿aún tenéis tortita o qué? }\end{array}$} \\
\hline Melodía & X (agudo) & \\
\hline Énfasis & $\mathrm{X}(\mathrm{yy})$ & \\
\hline Intensidad & & X (alta) \\
\hline \multicolumn{3}{|l|}{ Velocidad de habla } \\
\hline Pausas & & $\mathrm{X}$ \\
\hline \multicolumn{3}{|l|}{ Cualidad de la voz } \\
\hline Vocalizaciones no verbales & X (uff) & $\mathrm{X}$ (uff) \\
\hline \multicolumn{3}{|l|}{ Excitación } \\
\hline \multicolumn{3}{|l|}{$\begin{array}{l}\text { 490B: sí/ peroo- ehta mihma/ que se saque el carnée// y se ehté un año } \\
\text { sinÆ/ como hizo su madre/ ¿iqué hizo tu madre!?f/ sacarse el carné ((y } \\
\text { ehtarse doh o treh añoh sin)) coche/ y cuando se loo cogió el coche/ poh } \\
\text { un miedo que pa(ra) qué }\end{array}$} \\
\hline Melodía & $\mathrm{X}$ (agudo) & $\mathrm{X}$ (agudo) \\
\hline Énfasis & $\mathrm{X}($ miedo) & $\mathrm{x}$ (miedo) \\
\hline Intensidad & alta & alta \\
\hline Velocidad de habla & X (rápida) & X (rápida) \\
\hline Pausas & $\mathrm{X}$ & $\mathrm{X}$ \\
\hline \multicolumn{3}{|l|}{ Cualidad de la voz } \\
\hline \multicolumn{3}{|l|}{ Vocalizaciones no verbales } \\
\hline Excitación & $\mathrm{X}$ & $\mathrm{X}$ \\
\hline $\begin{array}{l}\text { 499B: una vez que ehtéih acostumbráh ya } \uparrow \text { / aunque lo ehtéih } \sin \rightarrow \\
\text { cogerlo/ pero de primerah } \uparrow \text { poh quee no/ porque muchah veceh se v }\end{array}$ & & \\
\hline
\end{tabular}




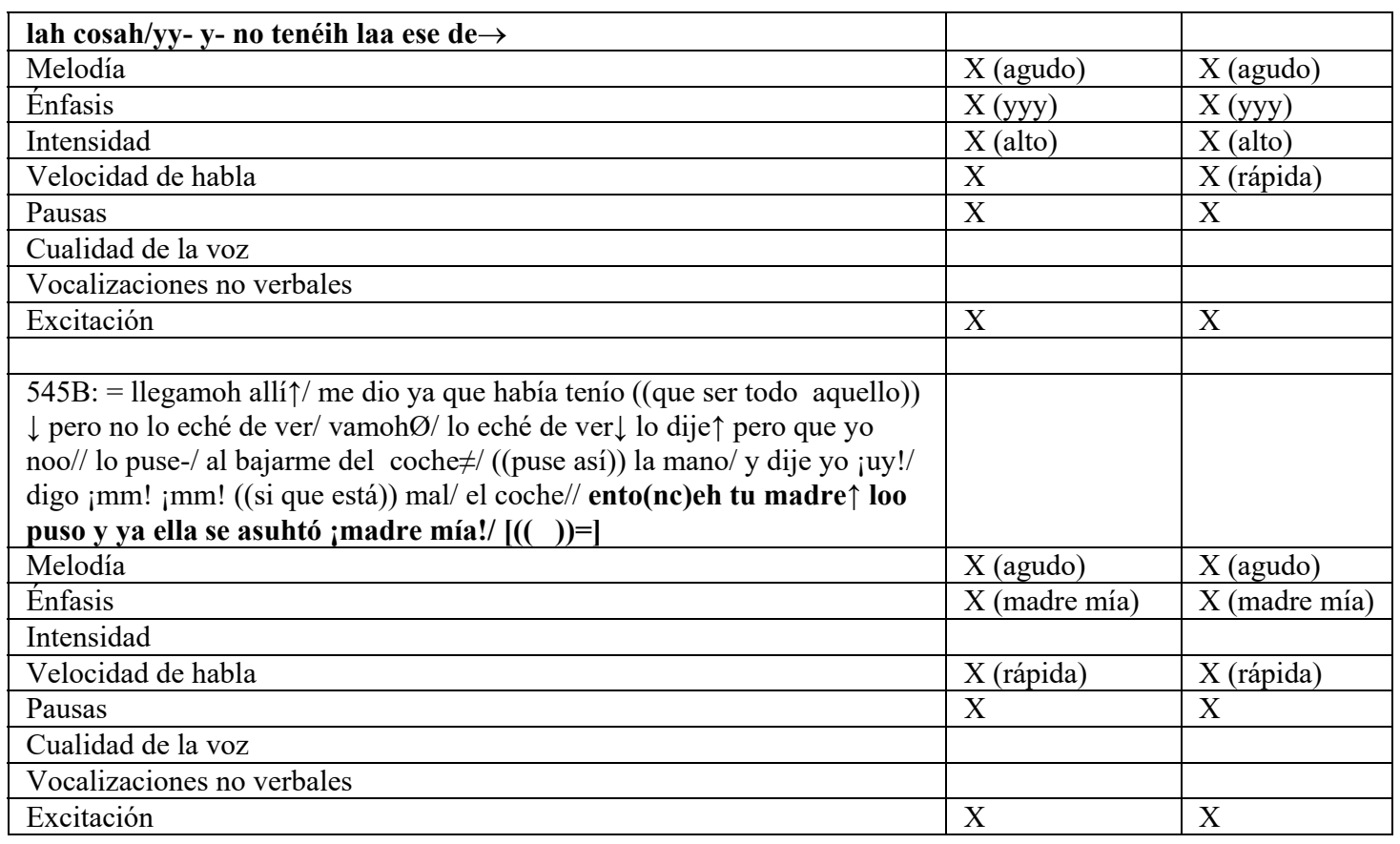

\begin{tabular}{|c|c|c|}
\hline TRISTEZA (7) & Observador1 & Observador2 \\
\hline \multicolumn{3}{|c|}{ 69B: '(pos que ya $\uparrow /$ no $\downarrow / / /$ ique se lo hubieran echao a otro!) ${ }^{\circ}\left(3^{\prime \prime}\right)$} \\
\hline Melodía & $\mathrm{X}$ (grave) & $\mathrm{X}$ (grave) \\
\hline \multicolumn{3}{|l|}{ Énfasis } \\
\hline Intensidad & $\mathrm{X}$ (bajo) & $\mathrm{X}$ (bajo) \\
\hline Velocidad de habla & X (lenta) & X (lenta) \\
\hline Pausas & $\mathrm{X}$ & $\mathrm{X}$ \\
\hline \multicolumn{3}{|l|}{ Cualidad de la voz } \\
\hline \multicolumn{3}{|c|}{ Vocalizaciones no verbales } \\
\hline Excitación & & $\mathrm{X}$ \\
\hline \multicolumn{3}{|c|}{ 178B: $\quad$ \& yo no } \\
\hline Melodía & $\mathrm{X}$ (grave) & $\mathrm{X}$ (grave) \\
\hline \multicolumn{3}{|l|}{ Énfasis } \\
\hline \multicolumn{3}{|l|}{ Intensidad } \\
\hline Velocidad de habla & $\mathrm{X}$ (lenta) & $\mathrm{X}$ (lenta) \\
\hline \multicolumn{3}{|l|}{ Pausas } \\
\hline \multicolumn{3}{|l|}{ Cualidad de la voz } \\
\hline \multicolumn{3}{|c|}{ Vocalizaciones no verbales } \\
\hline \multicolumn{3}{|l|}{ Excitación } \\
\hline \multicolumn{3}{|c|}{$\begin{array}{l}\text { 180B: no me he enterao de na(da)/ de na(da) de na(da)/ na(da) más } \\
\text { [quee] }\end{array}$} \\
\hline Melodía & $\mathrm{X}$ (grave) & \\
\hline Énfasis & $X(n a)$ & \\
\hline Intensidad & & X (alta) \\
\hline Velocidad de habla & $\mathrm{X}$ (rápida) & \\
\hline Pausas & $\mathrm{X}$ & $\mathrm{X}$ \\
\hline \multicolumn{3}{|l|}{ Cualidad de la voz } \\
\hline \multicolumn{3}{|c|}{ Vocalizaciones no verbales } \\
\hline Excitación & & $\mathrm{X}$ \\
\hline \multicolumn{3}{|c|}{$\begin{array}{l}183 \text { B: dende el día que fuimoh nosotroh y la vimoh } \uparrow / \text { yo no me he } \\
\left.\text { enterao de na(da) } /^{\circ}{ }^{\circ} \text { (ni he vihto a nadie }\right)^{\circ}\end{array}$} \\
\hline Melodía & $\mathrm{X}$ (grave) & $\mathrm{X}$ (grave) \\
\hline Énfasis & $\mathrm{X}$ & $\mathrm{X}$ \\
\hline Intensidad & X (baja) & X (baja) \\
\hline Velocidad de habla & X (lenta) & $\mathrm{X}$ (rápida) \\
\hline Pausas & $\mathrm{X}$ & $\mathrm{X}$ \\
\hline
\end{tabular}




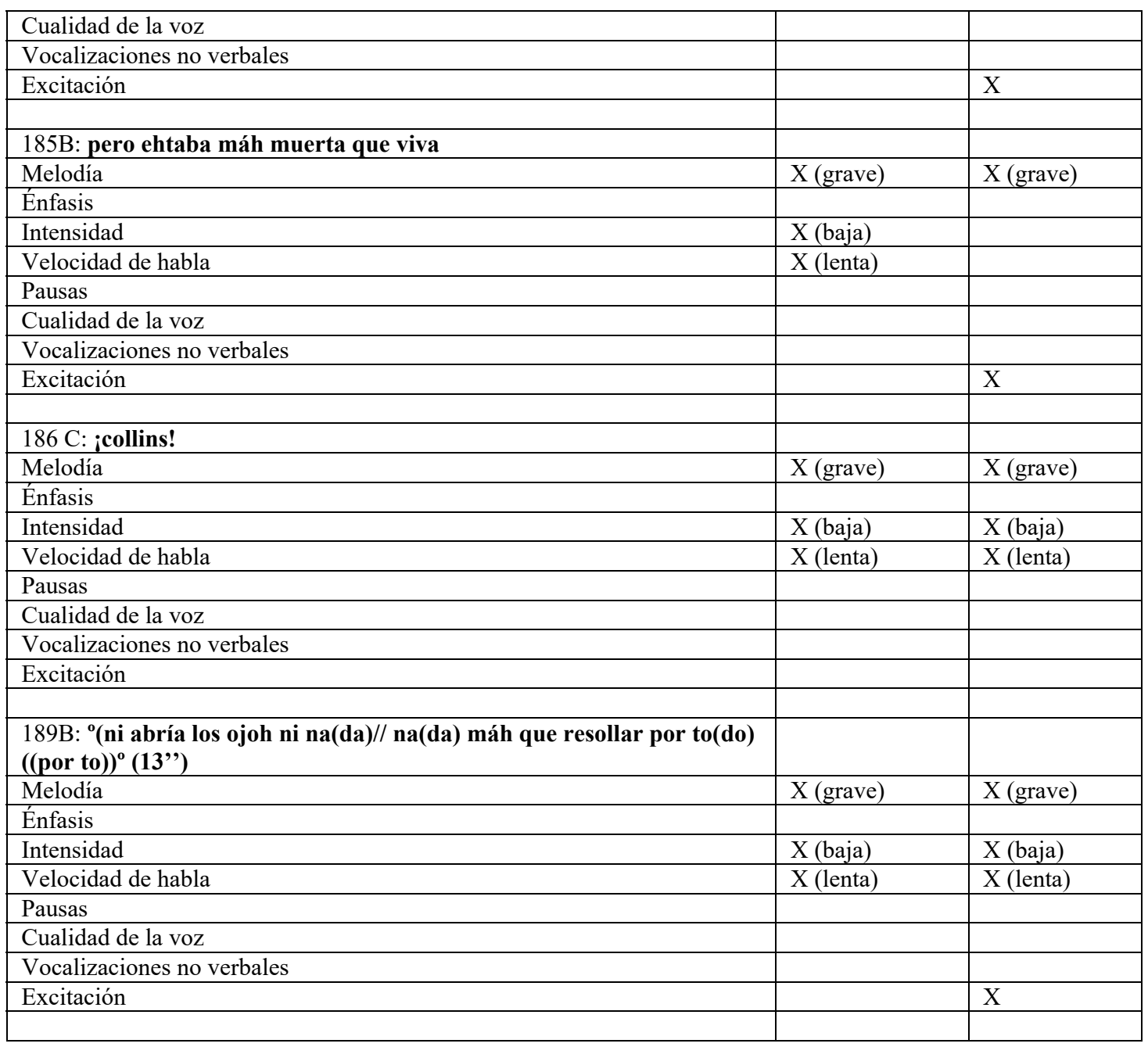

\begin{tabular}{|c|c|c|}
\hline ENFADO (7) & Observador1 & Observador2 \\
\hline \multicolumn{3}{|l|}{$\begin{array}{l}\text { 32A: } \quad \S((\text { deja })) / \text { estáte quieta§ } \\
\text { 34A: } \S \text { que está ella leyendo }\end{array}$} \\
\hline Melodía & $\mathrm{X}$ (grave) & \\
\hline \multicolumn{3}{|l|}{ Énfasis } \\
\hline Intensidad & $\mathrm{X}$ (alto) & $\mathrm{X}$ (alto) \\
\hline Velocidad de habla & X (rápida) & X (rápida) \\
\hline Pausas & $X$ & \\
\hline \multicolumn{3}{|l|}{ Cualidad de la voz } \\
\hline \multicolumn{3}{|l|}{ Vocalizaciones no verbales } \\
\hline Excitación & & $\mathrm{X}$ \\
\hline \multicolumn{3}{|l|}{ 36B: porque si es verdad to(do) lo que dicen $\uparrow /$ ¡vaya una puñeta! (3’') } \\
\hline Melodía & X (aguda) & \\
\hline \multicolumn{3}{|l|}{ Énfasis } \\
\hline Intensidad & & $\mathrm{X}$ (alto) \\
\hline Velocidad de habla & $\mathrm{X}$ (rápida) & X (rápida) \\
\hline Pausas & $\mathrm{X}$ & \\
\hline \multicolumn{3}{|l|}{ Cualidad de la voz } \\
\hline \multicolumn{3}{|l|}{ Vocalizaciones no verbales } \\
\hline Excitación & & $\mathrm{X}$ \\
\hline $\begin{array}{l}\text { 406C: no/// ahora me toca otra vez las prácticas/ si apruebo quiero } \\
\text { quedarme una semanaa/ para probar/ alli/ porque resulta/ abuela/ que } \\
\text { aquí estoy en casa } \uparrow / / \text { YY- HM HAGO MÁS FAENA QUE LO QUE } \\
\text { HE HECHO§FUERA DE CASA } \downarrow / \text { QUE SI FREGAR } \rightarrow \text { / QUE si tengo } \\
\text { unas cosas por el MEDIO yy- no PUEDO }\end{array}$ & & \\
\hline
\end{tabular}




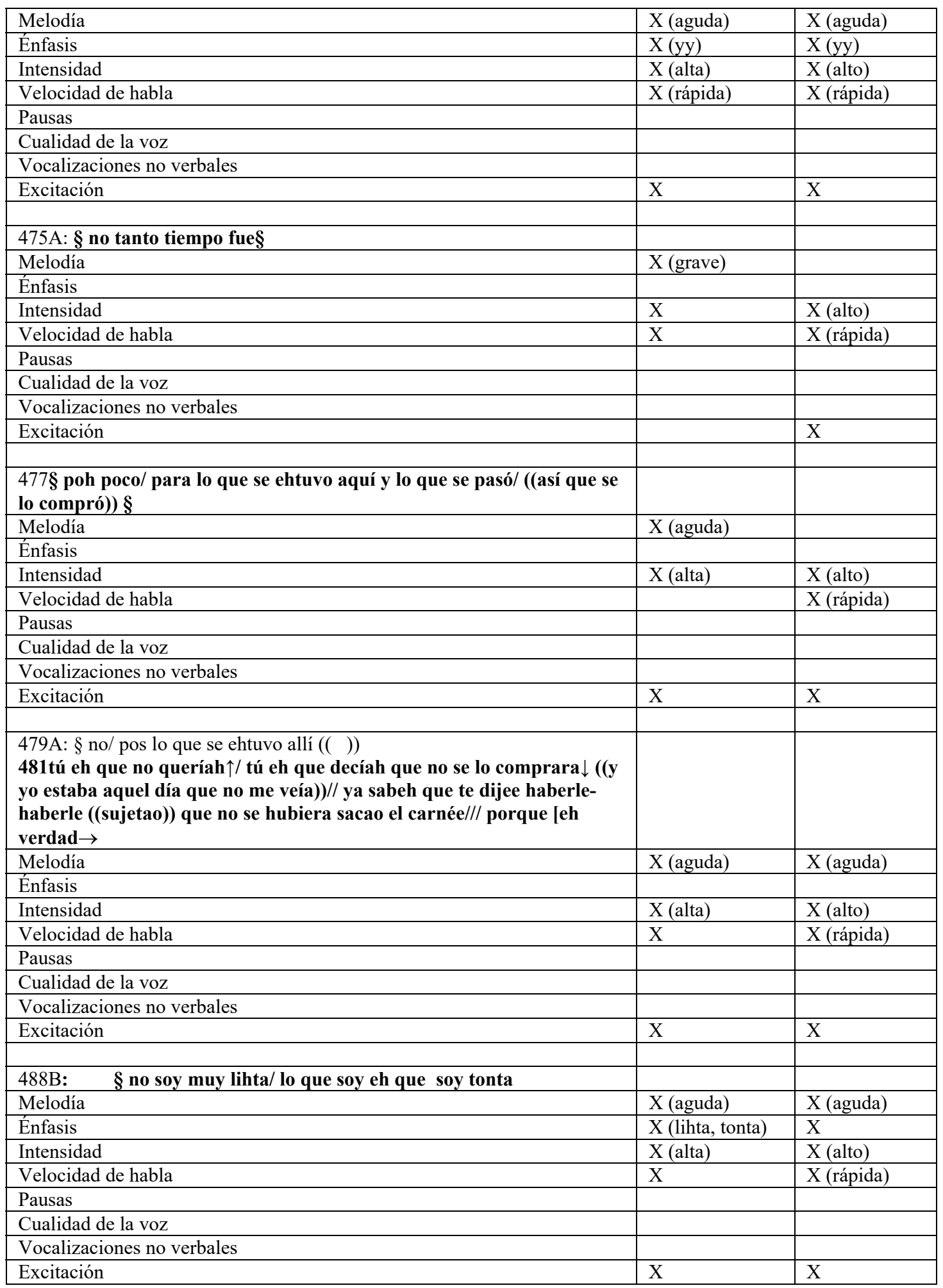

\begin{tabular}{|l|l|l|}
\hline SORPRESA (7) & Observador1 & Observador2 \\
\hline 12C: MI- A MI MADRE le enviaron una carta d'estas§ & & \\
\hline Melodía & X (aguda) & X (aguda) \\
\hline Énfasis & $\begin{array}{l}\text { X (MI- A MI } \\
\text { MADRE) }\end{array}$ & X \\
\hline Intensidad & X (alta) & X (alto) \\
\hline Velocidad de habla & X (rápida) & X (rápida) \\
\hline Pausas & & \\
\hline Cualidad de la voz & & \\
\hline
\end{tabular}




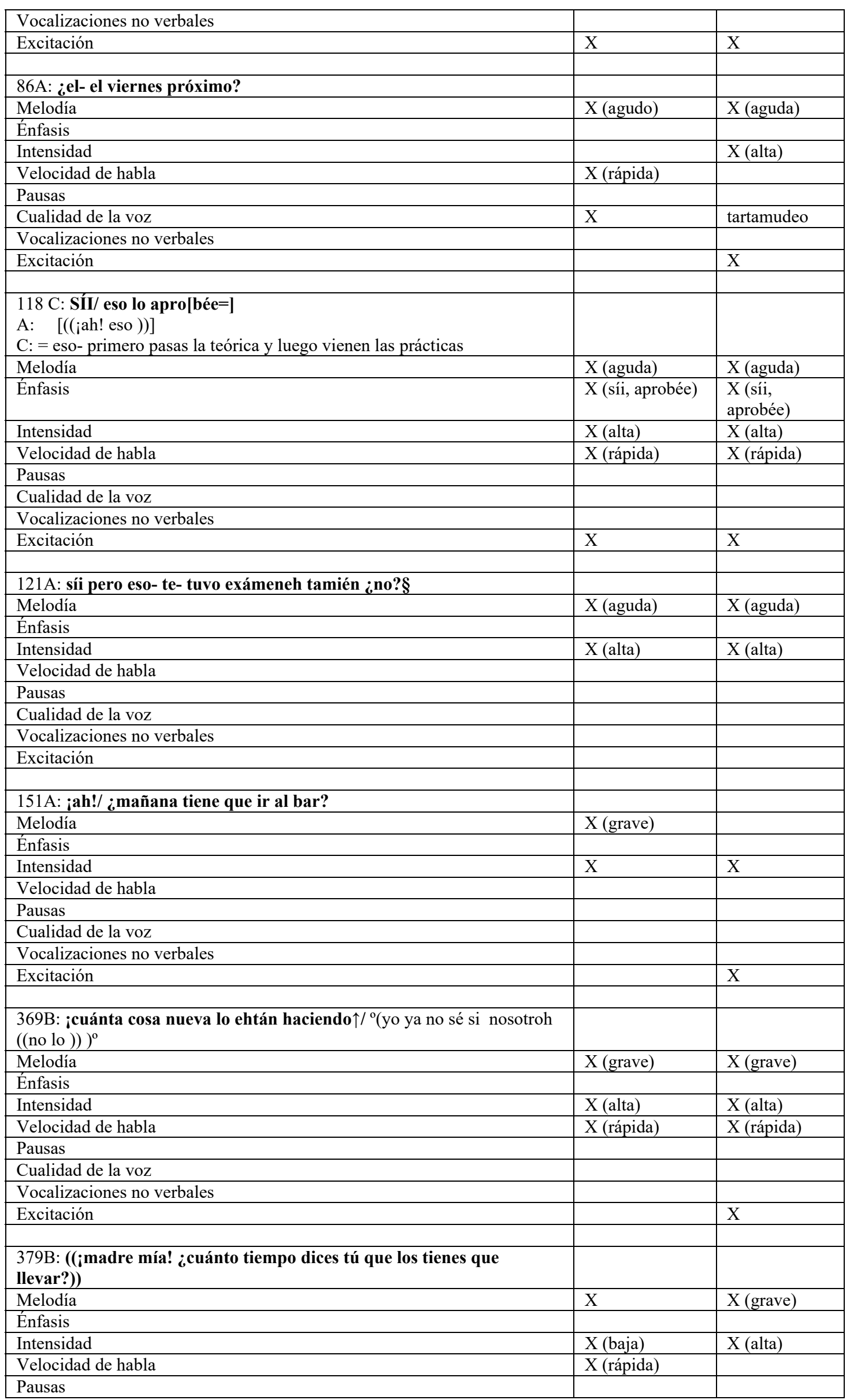


Cualidad de la voz

Vocalizaciones no verbales

Excitación

\begin{tabular}{|l|l|}
\hline & \\
\hline & $\mathrm{X}$ \\
\hline
\end{tabular}

\begin{tabular}{|c|c|c|}
\hline ALEGRÍA (7) & Observador1 & Observador2 \\
\hline \multicolumn{3}{|c|}{ 88C: (RISAS) ¡cómo se ríe la abuela! $§$} \\
\hline \multicolumn{3}{|l|}{ Melodía } \\
\hline \multicolumn{3}{|l|}{ Énfasis } \\
\hline Intensidad & & $\mathrm{X}$ (baja) \\
\hline Velocidad de habla & $\mathrm{X}$ (rápida) & \\
\hline \multicolumn{3}{|l|}{ Pausas } \\
\hline \multicolumn{3}{|l|}{ Cualidad de la voz } \\
\hline Vocalizaciones no verbales & $\mathrm{X}$ (risas) & $\mathrm{X}$ (risas) \\
\hline \multicolumn{3}{|l|}{ Excitación } \\
\hline \multicolumn{3}{|c|}{ 100A: que eh vierneh/// iAYY// si te- ya le dan el carnée!§ } \\
\hline Melodía & $\mathrm{X}$ (agudo) & $\mathrm{X}$ (agudo) \\
\hline Énfasis & $\mathrm{X}($ ¡AYY!) & $\mathrm{X}($ ¡AYY!) \\
\hline \multicolumn{3}{|l|}{ Intensidad } \\
\hline Velocidad de habla & X (rápida) & X (rápida) \\
\hline \multicolumn{3}{|l|}{ Pausas } \\
\hline \multicolumn{3}{|l|}{ Cualidad de la voz } \\
\hline Vocalizaciones no verbales & ayy & ayy \\
\hline Excitación & $\mathrm{X}$ & $\mathrm{X}$ \\
\hline \multicolumn{3}{|c|}{ 125A: ¿y lo aprobasteh todo?/ ¡ayy/ qué lista es!§ } \\
\hline Melodía & $\mathrm{X}$ & $\mathrm{X}$ \\
\hline & $\mathrm{X}$ (lis-) & $\mathrm{X}($ ¡AYY!) \\
\hline \multicolumn{3}{|l|}{$\begin{array}{l}\text { Enfasis } \\
\text { Intensidad }\end{array}$} \\
\hline Velocidad de habla & & $\mathrm{X}$ (rápida) \\
\hline \multicolumn{3}{|l|}{ Pausas } \\
\hline \multicolumn{3}{|l|}{ Cualidad de la voz } \\
\hline Vocalizaciones no verbales & $\mathrm{X}(\mathrm{AYY})$ & ayy \\
\hline \multicolumn{3}{|l|}{ Excitación } \\
\hline \multicolumn{3}{|c|}{ 321C: QUE ESTÁS CAVILOSO/ ABUELO } \\
\hline Melodía & $\mathrm{X}$ & $\mathrm{X}$ (agudo) \\
\hline \multicolumn{3}{|l|}{ Énfasis } \\
\hline Intensidad & X (alta) & $\mathrm{X}$ (alta) \\
\hline Velocidad de habla & $\mathrm{X}$ (rápida) & X (rápida) \\
\hline \multicolumn{3}{|l|}{ Pausas } \\
\hline \multicolumn{3}{|l|}{ Cualidad de la voz } \\
\hline \multicolumn{3}{|l|}{ Vocalizaciones no verbales } \\
\hline \multicolumn{3}{|l|}{ Excitación } \\
\hline 349B: toma valientass & & \\
\hline Melodía & $\mathrm{X}$ (agudo) & $\mathrm{X}$ \\
\hline Énfasis & $\mathrm{X}$ (tass) & $\mathrm{X}$ \\
\hline Intensidad & X (alta) & X (alta) \\
\hline Velocidad de habla & & \\
\hline Pausas & & \\
\hline Cualidad de la voz & & \\
\hline Vocalizaciones no verbales & & \\
\hline Excitación & & $\mathrm{X}$ \\
\hline 350C: ¡ah! ¿eso lo compras & & \\
\hline Melodía & $\mathrm{X}$ (agudo) & $\mathrm{X}$ (agudo) \\
\hline Énfasis & & \\
\hline Intensidad & X (alta) & X (alta) \\
\hline Velocidad de habla & X (rápida) & $\mathrm{X}$ \\
\hline Pausas & & \\
\hline Cualidad de la voz & & \\
\hline Vocalizaciones no verbales & & \\
\hline
\end{tabular}




\begin{tabular}{|l|l|l|}
\hline Excitación & X & X \\
\hline 489A: no/ puehentonceh $\rightarrow$ & & \\
\hline Melodía & & \\
\hline Énfasis & $\mathrm{X}$ & \\
\hline Intensidad & & $\mathrm{X}$ (alta) \\
\hline Velocidad de habla & & $\mathrm{X}$ (rápida) \\
\hline Pausas & & \\
\hline Cualidad de la voz & & \\
\hline Vocalizaciones no verbales & $\mathrm{X}$ (entre risas) & $\mathrm{X}$ \\
\hline Excitación & & $\mathrm{X}$ \\
\hline
\end{tabular}

\begin{tabular}{|l|l|l|}
\hline ASCO (1) & Observador1 & Observador2 \\
\hline $\begin{array}{l}\text { 354C: no/ no quiero/ abuela/ de verdad// si ahora comería } \uparrow / \text { a(ho)ra } \\
\text { que estoy/ me (...)/ ihace un frío por la calle!/ imadre mía! }\end{array}$ & & \\
\hline Melodía & $\mathrm{X}$ & $\mathrm{X}$ \\
\hline Énfasis & $\mathrm{X}(\mathrm{no} / \mathrm{no})$ & \\
\hline Intensidad & & \\
\hline Velocidad de habla & $\mathrm{X}$ & $\mathrm{X}$ \\
\hline Pausas & $\mathrm{X}$ & $\mathrm{X}$ \\
\hline Cualidad de la voz & & \\
\hline Vocalizaciones no verbales & & \\
\hline Excitación & & \\
\hline
\end{tabular}

\section{APÉNDICE 2}

\section{Emoción $=$ Miedo}

\section{Tabla cruzada Observador $2 *$ Observador $1^{a}$}

Recuento

\begin{tabular}{|c|c|c|c|c|}
\hline & & \multicolumn{2}{|c|}{ Observador1 } & \multirow[b]{2}{*}{ Total } \\
\hline & & ausente & presente & \\
\hline \multirow[t]{2}{*}{ Observador2 } & ausente & 20 & 5 & 25 \\
\hline & presente & 4 & 27 & 31 \\
\hline Total & & 24 & 32 & 56 \\
\hline
\end{tabular}

a. Emoción $=$ Miedo

\section{Medidas simétricas ${ }^{\mathrm{a}}$}

\begin{tabular}{l|l|l|l|} 
Valor & Error estándar asintótico & T & aproximada \\
& & Significación aproximada \\
\hline
\end{tabular}

\begin{tabular}{lrr|r|r|r}
\hline Medida de acuerdo & Kappa &, 674 &, 100 & 5,044 &, 000 \\
\hline N de casos válidos & 56 & & & \\
\hline
\end{tabular}
a. Emoción $=$ Miedo
b. No se presupone la hipótesis nula.
c. Utilización del error estándar asintótico que presupone la hipótesis nula.

\section{Emoción $=$ Tristeza}

Tabla cruzada Observador $2 *$ Observador $1^{\text {a }}$

Recuento

\begin{tabular}{|c|c|c|c|c|}
\hline & & \multicolumn{2}{|c|}{ Observador1 } & \multirow[b]{2}{*}{ Total } \\
\hline & & ausente & presente & \\
\hline \multirow[t]{2}{*}{ Observador2 } & ausente & 26 & 5 & 31 \\
\hline & presente & 5 & 20 & 25 \\
\hline \multicolumn{2}{|l|}{ Total } & 31 & 25 & 56 \\
\hline
\end{tabular}

a. Emoción $=$ Tristeza

Medidas simétricas ${ }^{\mathrm{a}}$

\begin{tabular}{c|c|c|c|c|} 
Valor & Error estándar asintótico & T & aproximada & \\
& & Significación aproximada \\
\hline
\end{tabular}

\begin{tabular}{lr|r|r|r}
\hline Medida de acuerdo Kappa &, 639 &, 103 & 4,780 &, 000 \\
\hline N de casos válidos & 56 & & & \\
\hline
\end{tabular}


a. Emoción $=$ Tristeza

b. No se presupone la hipótesis nula.

c. Utilización del error estándar asintótico que presupone la hipótesis nula.

\section{Emoción $=$ Enfado}

\section{Tabla cruzada Observador $2 *$ Observador $1^{a}$}

Recuento

\begin{tabular}{|c|c|c|c|c|}
\hline & & \multicolumn{2}{|c|}{ Observador1 } & Total \\
\hline \multirow[t]{2}{*}{ Observador2 } & ausente & 23 & 6 & 29 \\
\hline & presente & 5 & 22 & 27 \\
\hline Total & & 28 & 28 & 56 \\
\hline
\end{tabular}

a. Emoción $=$ Enfado

Medidas simétricas ${ }^{\mathbf{a}}$

\begin{tabular}{c|c|c|c|} 
Valor Error estándar asintótico & $\mathrm{T}$ aproximada & Significación aproximada $^{\mathrm{c}}$ & Sign
\end{tabular}

\begin{tabular}{|c|c|c|c|c|}
\hline Medida de acuerdo Kappa & ,607 & 106 & 4,546 & 000 \\
\hline $\mathrm{N}$ de casos válidos & 56 & & & \\
\hline
\end{tabular}

a. Emoción $=$ Enfado

b. No se presupone la hipótesis nula.

c. Utilización del error estándar asintótico que presupone la hipótesis nula.

\section{Emoción $=$ Sorpresa}

Tabla cruzada Observador $2 *$ Observador $1^{a}$

Recuento

\begin{tabular}{|c|c|c|c|c|}
\hline & & \multicolumn{2}{|c|}{ Observador1 } & \multirow[b]{2}{*}{ Total } \\
\hline & & ausente & presente & \\
\hline \multirow[t]{2}{*}{ Observador2 } & ausente & 28 & 2 & 30 \\
\hline & presente & 5 & 21 & 26 \\
\hline Total & & 33 & 23 & 56 \\
\hline
\end{tabular}

a. Emoción $=$ Sorpresa

Medidas simétricas ${ }^{\mathrm{a}}$

\begin{tabular}{l|l|l|l|} 
Valor & Error estándar asintótico & & $\mathrm{T}$ aproximada \\
& & Significación aproximada \\
\hline
\end{tabular}

\begin{tabular}{lrr|r|r|r}
\hline Medida de acuerdo & Kappa &, 747 &, 089 & 5,622 &, 000 \\
\hline N de casos válidos & 56 & & & \\
\hline
\end{tabular}

a. Emoción $=$ Sorpresa

b. No se presupone la hipótesis nula.

c. Utilización del error estándar asintótico que presupone la hipótesis nula.

\section{Emoción = Alegría}

\section{Tabla cruzada Observador $2 *$ Observador $1^{a}$}

Recuento

\begin{tabular}{ll|r|r|r} 
& & \multicolumn{2}{c|}{ Observador1 } & \\
& & ausente & presente & Total \\
\hline Observador2 & ausente & 26 & 2 & 28 \\
\cline { 2 - 6 } & presente & 8 & 20 & 28 \\
\hline Total & & 34 & 22 & 56 \\
\hline
\end{tabular}

a. Emoción = Alegría

Medidas simétricas ${ }^{\mathrm{a}}$

\begin{tabular}{l|l|l|l|} 
Valor & Error estándar asintótico & $\mathrm{T}$ aproximada & Significación aproximada $^{\mathrm{c}}$
\end{tabular}

\begin{tabular}{|r|r|r|r|r|}
\hline Medida de acuerdo Kappa &, 643 &, 100 & 4,925 &, 000 \\
\hline N de casos válidos & 56 & & & \\
\hline
\end{tabular}

a. Emoción $=$ Alegría

b. No se presupone la hipótesis nula.

c. Utilización del error estándar asintótico que presupone la hipótesis nula. 


$\begin{aligned} & \text { Emoción = Asco } \\
& \text { Tabla cruzada Observador } 2 * \text { Observador }^{\mathbf{a}} \\
& \text { Recuento }\end{aligned}$
\begin{tabular}{llr|r|r} 
& \multicolumn{2}{l}{$\begin{array}{l}\text { Observador } 1 \\
\text { ausente }\end{array}$} & presente & Total \\
\hline Observador2 & ausente & 4 & 1 & 5 \\
\cline { 2 - 5 } & presente & 0 & 3 & 3 \\
\hline Total & 4 & 4 & 8 \\
\hline
\end{tabular}

a. Emoción $=$ Asco

Medidas simétricas ${ }^{\mathrm{a}}$

\begin{tabular}{l|l|l|l} 
Valor & Error estándar asintótico & $\mathrm{T}$ aproximada & Significación aproximada $^{\mathrm{c}}$ \\
\hline
\end{tabular}

\begin{tabular}{lr|r|r|r|r}
\hline Medida de acuerdo Kappa &, 750 &, 226 & 2,191 &, 028 \\
\hline $\mathrm{N}$ de casos válidos & 8 & & & \\
\hline
\end{tabular}

a. Emoción $=$ Asco

b. No se presupone la hipótesis nula.

c. Utilización del error estándar asintótico que presupone la hipótesis nula.

\section{APÉNDICE 3}

\section{Rasgo $=$ Melodía}

Tabla cruzada Observador $2 *$ Observador $1^{a}$

Recuento

\begin{tabular}{|c|c|c|c|c|}
\hline & & \multicolumn{2}{|c|}{ Observador1 } & \multirow[b]{2}{*}{ Total } \\
\hline & & ausente & presente & \\
\hline \multirow{2}{*}{ Observador2 } & ausente & 1 & 9 & 10 \\
\hline & presente & 0 & 26 & 26 \\
\hline Total & & 1 & 35 & 36 \\
\hline
\end{tabular}

a. Rasgo $=$ Melodía

Medidas simétricas ${ }^{\mathrm{a}}$

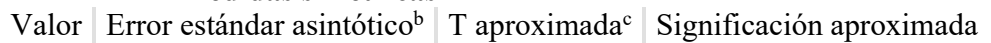

\begin{tabular}{lrr|r|r|r}
\hline Medida de acuerdo & Kappa &, 138 &, 126 & 1,635 &, 102 \\
\hline $\mathrm{N}$ de casos válidos & 36 & & & \\
\hline
\end{tabular}
a. Rasgo $=$ Melodía
b. No se presupone la hipótesis nula.
c. Utilización del error estándar asintótico que presupone la hipótesis nula.

\section{Rasgo $=$ Énfasis}

Tabla cruzada Observador $2 *$ Observador $1^{a}$

Recuento

\begin{tabular}{|c|c|c|c|c|}
\hline & & \multicolumn{2}{|c|}{ Observador1 } & \multirow[b]{2}{*}{ Total } \\
\hline & & ausente & presente & \\
\hline \multirow[t]{2}{*}{ Observador2 } & ausente & 19 & 4 & 23 \\
\hline & presente & 0 & 13 & 13 \\
\hline Total & & 19 & 17 & 36 \\
\hline
\end{tabular}

a. Rasgo $=$ Énfasis

\begin{tabular}{|c|c|c|c|c|}
\hline \multicolumn{5}{|c|}{ Medidas simétricas $^{\mathrm{a}}$} \\
\hline & Valor & Error estándar asintótico ${ }^{\mathrm{b}}$ & $\mathrm{T}$ aproximada ${ }^{\mathrm{c}}$ & Significación aproximada \\
\hline Medida de acuerdo Kappa & ,774 & ,104 & 4,769 & ,000 \\
\hline $\mathrm{N}$ de casos válidos & 36 & & & \\
\hline
\end{tabular}
a. Rasgo $=$ Énfasis
b. No se presupone la hipótesis nula.
c. Utilización del error estándar asintótico que presupone la hipótesis nula. 


\begin{tabular}{|c|c|c|c|c|}
\hline \multirow{2}{*}{\multicolumn{5}{|c|}{$\begin{array}{l}\text { Rasgo }=\text { Intensidad } \\
\text { Tabla cruzada Observador } 2 * \text { Observador }^{\text {a }} \\
\text { Recuento }\end{array}$}} \\
\hline & & & & \\
\hline & & \multicolumn{2}{|c|}{ Observador1 } & Total \\
\hline \multirow[t]{2}{*}{ Observador 2} & ausente & 4 & 1 & 5 \\
\hline & presente & 8 & 23 & 31 \\
\hline Total & & 12 & 24 & 36 \\
\hline
\end{tabular}

a. Rasgo $=$ Intensidad

Medidas simétricas ${ }^{\mathrm{a}}$

\begin{tabular}{l|l|l|l|} 
Valor & Error estándar asintótico & $\mathrm{T}$ aproximada $^{\mathrm{c}}$ & Significación aproximada $^{\mathrm{b}}$
\end{tabular}

\begin{tabular}{lr|r|r|r|r}
\hline Medida de acuerdo & Kappa &, 341 &, 157 & 2,385 &, 017 \\
\hline $\mathrm{N}$ de casos válidos & 36 & & & \\
\hline
\end{tabular}

a. Rasgo $=$ Intensidad

b. No se presupone la hipótesis nula.

c. Utilización del error estándar asintótico que presupone la hipótesis nula.

\section{Rasgo $=$ Velocidad}

Tabla cruzada Observador $2 *$ Observador $1^{a}$

Recuento

\begin{tabular}{|c|c|c|c|c|}
\hline & & \multicolumn{2}{|c|}{ Observador1 } & \multirow[b]{2}{*}{ Total } \\
\hline & & ausente & presente & \\
\hline \multirow[t]{2}{*}{ Observador2 } & ausente & 5 & 4 & 9 \\
\hline & presente & 3 & 24 & 27 \\
\hline Total & & 8 & 28 & 36 \\
\hline
\end{tabular}

a. Rasgo $=$ Velocidad

\section{Medidas simétricas ${ }^{a}$}

\begin{tabular}{l|l|l|l|l} 
Valor & Error estándar asintótico & T aproximada & & Significación aproximada \\
\hline
\end{tabular}

\begin{tabular}{lr|r|r|r|r}
\hline Medida de acuerdo & Kappa &, 462 &, 173 & 2,777 &, 005 \\
\hline N de casos válidos & 36 & & & \\
\hline
\end{tabular}

a. Rasgo $=$ Velocidad

b. No se presupone la hipótesis nula.

c. Utilización del error estándar asintótico que presupone la hipótesis nula.

\section{Rasgo $=$ Pausas}

\section{Tabla cruzada Observador $2 *$ Observador $1{ }^{a}$}

Recuento

\begin{tabular}{ll|r|r|r} 
& & \multicolumn{2}{c|}{ Observador1 } & \\
& & ausente & presente & Total \\
\hline Observador2 & ausente & 25 & 3 & 28 \\
\cline { 2 - 6 } & presente & 1 & 7 & 8 \\
\hline Total & & 26 & 10 & 36 \\
\hline
\end{tabular}

a. Rasgo $=$ Pausas

\section{Medidas simétricas ${ }^{\mathrm{a}}$}

\begin{tabular}{c|c|c|c|} 
Valor Error estándar asintótico & $\mathrm{T}$ aproximada & Significación aproximada $^{\mathrm{c}}$
\end{tabular}

\begin{tabular}{lr|r|r|r|r}
\hline Medida de acuerdo & Kappa &, 705 &, 136 & 4,276 &, 000 \\
\hline N de casos válidos & 36 & & & \\
\hline
\end{tabular}

a. Rasgo $=$ Pausas

b. No se presupone la hipótesis nula.

c. Utilización del error estándar asintótico que presupone la hipótesis nula.

\section{Rasgo $=$ Cualidad voz \\ Tabla cruzada Observador $2 *$ Observador $1{ }^{a}$}

Recuento 


\begin{tabular}{|c|c|c|c|c|}
\hline & & $\begin{array}{r}\text { Obse } \\
\text { ausente }\end{array}$ & $\begin{array}{l}\text { vador1 } \\
\text { presente }\end{array}$ & Total \\
\hline \multirow[t]{2}{*}{ Observador2 } & ausente & 35 & 0 & 35 \\
\hline & presente & 0 & 1 & 1 \\
\hline \multicolumn{2}{|l|}{ Total } & 35 & 1 & 36 \\
\hline
\end{tabular}

a. Rasgo $=$ Cualidad voz

Medidas simétricas ${ }^{\mathrm{a}}$

\begin{tabular}{l|l|l|l|} 
Valor & Error estándar asintótico & $\mathrm{T}$ aproximada & Significación aproximada $^{\mathrm{c}}$
\end{tabular}

\begin{tabular}{lrr|r|r|r}
\hline Medida de acuerdo & Kappa & 1,000 &, 000 & 6,000 &, 000 \\
\hline N de casos válidos & 36 & & & \\
\hline
\end{tabular}

a. Rasgo $=$ Cualidad voz

b. No se presupone la hipótesis nula.

c. Utilización del error estándar asintótico que presupone la hipótesis nula.

Rasgo $=$ no verbal

Tabla cruzada Observador $2 *$ Observador $1^{\mathrm{a}}$

Recuento

\begin{tabular}{|c|c|c|c|c|}
\hline & & \multicolumn{2}{|c|}{ Observador1 } & \multirow[b]{2}{*}{ Total } \\
\hline & & ausente & presente & \\
\hline \multirow[t]{2}{*}{ Observador2 } & ausente & 28 & 0 & 28 \\
\hline & presente & 0 & 8 & 8 \\
\hline Total & & 28 & 8 & 36 \\
\hline
\end{tabular}

a. Rasgo $=$ no verbal

Medidas simétricas ${ }^{a}$

\begin{tabular}{r|r|r|r|} 
Valor & Error estándar asintótico & T aproximada & \\
& & Significación aproximada $^{\mathrm{b}}$ &
\end{tabular}

\begin{tabular}{|rr|r|r|r}
\hline Medida de acuerdo Kappa & 1,000 &, 000 & 6,000 &, 000 \\
\hline N de casos válidos & 36 & & & \\
\hline
\end{tabular}

a. Rasgo $=$ no verbal

b. No se presupone la hipótesis nula.

c. Utilización del error estándar asintótico que presupone la hipótesis nula.

\section{Rasgo $=$ excitación}

\section{Tabla cruzada Observador $2 *$ Observador $1^{\text {a }}$}

Recuento

\begin{tabular}{llr|r|r} 
& & \multicolumn{2}{c|}{ Observador1 } & \\
& & ausente & presente & Total \\
\hline Observador2 & ausente & 10 & 0 & 10 \\
\cline { 2 - 5 } & presente & 15 & 11 & 26 \\
\hline Total & & 25 & 11 & 36 \\
\hline
\end{tabular}

a. Rasgo $=$ excitación

Medidas simétricas ${ }^{\mathrm{a}}$

\begin{tabular}{ll|l|l|l} 
Valor & Error estándar asintótico & $\mathrm{T}$ aproximada & Significación aproximada $^{\mathrm{c}}$ & Sign
\end{tabular}

\begin{tabular}{lrr|r|r|r}
\hline Medida de acuerdo & Kappa &, 289 &, 099 & 2,468 &, 014 \\
\hline N de casos válidos & 36 & & & \\
\hline
\end{tabular}

a. Rasgo $=$ excitación

b. No se presupone la hipótesis nula.

c. Utilización del error estándar asintótico que presupone la hipótesis nula. 